Dynamic Systems and Applications 30 (2021) No.9, 1479-1501

\title{
EXISTENCE RESULTS FOR A SELF-ADJOINT COUPLED SYSTEM OF NONLINEAR ORDINARY DIFFERENTIAL EQUATIONS WITH NONLOCAL NON-SEPARATED INTEGRAL BOUNDARY CONDITIONS
}

\author{
AHMED ALSAEDI ${ }^{a}$, AMAL ALMALKI $^{a}$, SOTIRIS K. NTOUYAS ${ }^{b, a}$, BASHIR AHMAD $^{a}$, \\ AND RAVI P. AGARWAL ${ }^{c, a}$ \\ ${ }^{a}$ Nonlinear Analysis and Applied Mathematics (NAAM)-Research Group, \\ Department of Mathematics, Faculty of Science, King Abdulaziz University, P.O. \\ Box 80203, Jeddah 21589, Saudi Arabia. \\ ${ }^{b}$ Department of Mathematics, University of Ioannina, 45110 Ioannina, Greece. \\ ${ }^{c}$ Department of Mathematics, Texas A\& M University, Kingsville, Texas \\ 78363-8202, USA.
}

\begin{abstract}
In this article, we develop the existence theory for a self-adjoint coupled system of nonlinear ordinary differential equations supplemented with nonlocal non-separated integral boundary conditions on an arbitrary domain. The two existence results depend on the Leray-Schauder alternative and Schauder fixed point theorem, while the uniqueness result relies on the Banach contraction mapping principle. For the illustration of the obtained result, we constructed several examples in the last section.
\end{abstract}

AMS (MOS) Subject Classification. 34B10, 34B15, 47H10.

\section{INTRODUCTION}

Consider the following self-adjoint coupled system of nonlinear second-order ordinary differential equations on an arbitrary domain complemented with nonlocal non-separated integral boundary conditions of the form:

$$
\left\{\begin{array}{l}
\left(p(t) u^{\prime}(t)\right)^{\prime}=\mu_{1} f(t, u(t), v(t)), t \in[a, b] \\
\left(q(t) v^{\prime}(t)\right)^{\prime}=\mu_{2} g(t, u(t), v(t)), t \in[a, b] \\
\alpha_{1} u(a)+\alpha_{2} u(b)=\lambda_{1} \int_{a}^{\eta} v(s) d s, \alpha_{3} u^{\prime}(a)+\alpha_{4} u^{\prime}(b)=\lambda_{2} \int_{a}^{\eta} v^{\prime}(s) d s \\
\beta_{1} v(a)+\beta_{2} v(b)=\lambda_{3} \int_{\xi}^{b} u(s) d s, \beta_{3} v^{\prime}(a)+\beta_{4} v^{\prime}(b)=\lambda_{4} \int_{\xi}^{b} u^{\prime}(s) d s
\end{array}\right.
$$

Received July 1, 2021 ISSN1056-2176(Print); ISSN 2693-5295 (online) $\$ 15.00$ C Dynamic Publishers, Inc. www.dynamicpublishers.org; https://doi.org/10.46719/dsa202130.09.06. 
where $f, g:[a, b] \times \mathbb{R} \times \mathbb{R} \rightarrow \mathbb{R}$ are given continuous functions, $a<\eta<\xi<b$, $p, q \in C\left([a, b], \mathbb{R}^{+}\right), \alpha_{i}, \beta_{i}, \lambda_{i} \in \mathbb{R}^{+}, i=1,2,3,4, \mu_{j} \in \mathbb{R}^{+}, j=1,2$.

Let us now dwell on the importance of boundary value problems. These problems arise in the mathematical modeling of many real-world problems, for example, see $[23,3]$ and the references cited therein. This led to the development of many aspects of boundary value problems, such as, existence and stability of solutions, analytic and numerical methods for solving these problems, etc. Classical boundary conditions fail to formulate the changes happening in a given phenomena at arbitrary interior positions of the given domain. In order to cope with this situation, the concept of nonlocal boundary value conditions was introduced. For a detailed description of nonlocal boundary value problems, we refer the reader to the articles $[11,14,13,12$, $7,16,5,22,9,21,19,8,15,1,2]$.

Indeed the class of self-adjoint (Sturm-Liouville) boundary value problems constitutes an interesting area of research due to the occurrence of such problems in applied sciences, for instance, see $[4,6,18,20]$ and the references cited therein. It has been observed that the literature dealing with these problems is scarce and needs attention. This motivated us to introduce and investigate the existence criteria for the solutions of the problem (1.1).

The paper is arranged as follows. In Section 2, we present an auxiliary lemma which is pivotal to define the solution of problem (1.1). Section 3 contains the main existence and uniqueness results for the problem. In Section 4, some illustrative examples are constructed.

\section{AN AUXILIARY LEMMA}

In the following, we prove an auxiliary lemma which is basic in the study of the problem (1.1).

Lemma 2.1. For $f_{1}, g_{1} \in C([a, b], \mathbb{R})$ and $R \neq 0, E \neq 0$, the solution of the linear system

$$
\left\{\begin{array}{l}
\left(p(t) u^{\prime}(t)\right)^{\prime}=\mu_{1} f_{1}(t), t \in[a, b] \\
\left(q(t) v^{\prime}(t)\right)^{\prime}=\mu_{2} g_{1}(t), t \in[a, b] \\
\alpha_{1} u(a)+\alpha_{2} u(b)=\lambda_{1} \int_{a}^{\eta} v(s) d s, \alpha_{3} u^{\prime}(a)+\alpha_{4} u^{\prime}(b)=\lambda_{2} \int_{a}^{\eta} v^{\prime}(s) d s \\
\beta_{1} v(a)+\beta_{2} v(b)=\lambda_{3} \int_{\xi}^{b} u(s) d s, \beta_{3} v^{\prime}(a)+\beta_{4} v^{\prime}(b)=\lambda_{4} \int_{\xi}^{b} u^{\prime}(s) d s
\end{array}\right.
$$

can be expressed by the formulas:

$$
u(t)=\int_{a}^{t}\left(\frac{\mu_{1}}{p(u)} \int_{a}^{u} f_{1}(z) d z\right) d u+\frac{1}{R}\left[-\alpha_{2}\left(\beta_{1}+\beta_{2}\right) \int_{a}^{b}\left(\frac{\mu_{1}}{p(u)} \int_{a}^{u} f_{1}(z) d z\right) d u\right.
$$


$+\lambda_{1}\left(\beta_{1}+\beta_{2}\right) \int_{a}^{\eta} \int_{a}^{s}\left(\frac{\mu_{2}}{q(u)} \int_{a}^{u} g_{1}(z) d z\right) d u d s$

$-\lambda_{1} \beta_{2}(\eta-a) \int_{a}^{b}\left(\frac{\mu_{2}}{q(u)} \int_{a}^{u} g_{1}(z) d z\right) d u$

$\left.+\lambda_{1} \lambda_{3}(\eta-a) \int_{\xi}^{b} \int_{a}^{s}\left(\frac{\mu_{1}}{p(u)} \int_{a}^{u} f_{1}(z) d z\right) d u d s\right]$

$+\frac{1}{E R}\left[\left(E_{4} \alpha_{2}\left(\beta_{1}+\beta_{2}\right) \int_{a}^{b} \frac{1}{p(z)} d z-E_{3} \lambda_{1}\left(\beta_{1}+\beta_{2}\right) \int_{a}^{\eta} \int_{a}^{s} \frac{1}{q(z)} d z d s\right.\right.$

$+E_{3} \lambda_{1} \beta_{2}(\eta-a) \int_{a}^{b} \frac{1}{q(z)} d z-E_{4} \lambda_{1} \lambda_{3}(\eta-a) \int_{\xi}^{b} \int_{a}^{s} \frac{1}{p(z)} d z d s$

$\left.-R E_{4} \int_{a}^{t} \frac{1}{p(z)} d z\right)\left(\frac{\alpha_{4} \mu_{1}}{p(b)} \int_{a}^{b} f_{1}(z) d z\right)+\left(-E_{4} \alpha_{2}\left(\beta_{1}+\beta_{2}\right) \int_{a}^{b} \frac{1}{p(z)} d z\right.$

$+E_{3} \lambda_{1}\left(\beta_{1}+\beta_{2}\right) \int_{a}^{\eta} \int_{a}^{s} \frac{1}{q(z)} d z d s-E_{3} \lambda_{1} \beta_{2}(\eta-a) \int_{a}^{b} \frac{1}{q(z)} d z$

$\left.+E_{4} \lambda_{1} \lambda_{3}(\eta-a) \int_{\xi}^{b} \int_{a}^{s} \frac{1}{p(z)} d z d s+R E_{4} \int_{a}^{t} \frac{1}{p(z)} d z\right)\left(\int_{a}^{\eta} \frac{\lambda_{2} \mu_{2}}{q(s)} \int_{a}^{s} g_{1}(z) d z d s\right)$

$+\left(E_{2} \alpha_{2}\left(\beta_{1}+\beta_{2}\right) \int_{a}^{b} \frac{1}{p(z)} d z-E_{1} \lambda_{1}\left(\beta_{1}+\beta_{2}\right) \int_{a}^{\eta} \int_{a}^{s} \frac{1}{q(z)} d z d s\right.$

$+E_{1} \lambda_{1} \beta_{2}(\eta-a) \int_{a}^{b} \frac{1}{q(z)} d z-E_{2} \lambda_{1} \lambda_{3}(\eta-a) \int_{\xi}^{b} \int_{a}^{s} \frac{1}{p(z)} d z d s$

$\left.-R E_{2} \int_{a}^{t} \frac{1}{p(z)} d z\right)\left(\frac{\beta_{4} \mu_{2}}{q(b)} \int_{a}^{b} g_{1}(z) d z\right)+\left(-E_{2} \alpha_{2}\left(\beta_{1}+\beta_{2}\right) \int_{a}^{b} \frac{1}{p(z)} d z\right.$

$+E_{1} \lambda_{1}\left(\beta_{1}+\beta_{2}\right) \int_{a}^{\eta} \int_{a}^{s} \frac{1}{q(z)} d z d s-E_{1} \lambda_{1} \beta_{2}(\eta-a) \int_{a}^{b} \frac{1}{q(z)} d z$

$+E_{2} \lambda_{1} \lambda_{3}(\eta-a) \int_{\xi}^{b} \int_{a}^{s} \frac{1}{p(z)} d z d s$

$\left.\left.+R E_{2} \int_{a}^{t} \frac{1}{p(z)} d z\right)\left(\int_{\xi}^{b} \frac{\lambda_{4} \mu_{1}}{p(s)} \int_{a}^{s} f_{1}(z) d z d s\right)\right]$,

and

$$
\begin{aligned}
v(t)= & \int_{a}^{t}\left(\frac{\mu_{2}}{q(u)} \int_{a}^{u} g_{1}(z) d z\right) d u+\frac{1}{R}\left[-\alpha_{2} \lambda_{3}(b-\xi) \int_{a}^{b}\left(\frac{\mu_{1}}{p(u)} \int_{a}^{u} f_{1}(z) d z\right) d u\right. \\
& +\lambda_{1} \lambda_{3}(b-\xi) \int_{a}^{\eta} \int_{a}^{s}\left(\frac{\mu_{2}}{q(u)} \int_{a}^{u} g_{1}(z) d z\right) d u d s \\
& -\beta_{2}\left(\alpha_{1}+\alpha_{2}\right) \int_{a}^{b}\left(\frac{\mu_{2}}{q(u)} \int_{a}^{u} g_{1}(z) d z\right) d u \\
& \left.+\lambda_{3}\left(\alpha_{1}+\alpha_{2}\right) \int_{\xi}^{b} \int_{a}^{s}\left(\frac{\mu_{1}}{p(u)} \int_{a}^{u} f_{1}(z) d z\right) d u d s\right] \\
& +\frac{1}{E R}\left[\left(E_{4} \alpha_{2} \lambda_{3}(b-\xi) \int_{a}^{b} \frac{1}{p(z)} d z-E_{3} \lambda_{1} \lambda_{3}(b-\xi) \int_{a}^{\eta} \int_{a}^{s} \frac{1}{q(z)} d z d s\right.\right.
\end{aligned}
$$




$$
\begin{aligned}
& +E_{3} \beta_{2}\left(\alpha_{1}+\alpha_{2}\right) \int_{a}^{b} \frac{1}{q(z)} d z-E_{4} \lambda_{3}\left(\alpha_{1}+\alpha_{2}\right) \int_{\xi}^{b} \int_{a}^{s} \frac{1}{p(z)} d z d s \\
& \left.-R E_{3} \int_{a}^{t} \frac{1}{p(z)} d z\right)\left(\frac{\alpha_{4} \mu_{1}}{p(b)} \int_{a}^{b} f_{1}(z) d z\right)+\left(-E_{4} \alpha_{2} \lambda_{3}(b-\xi) \int_{a}^{b} \frac{1}{p(z)} d z\right. \\
& +E_{3} \lambda_{1} \lambda_{3}(b-\xi) \int_{a}^{\eta} \int_{a}^{s} \frac{1}{q(z)} d z d s-E_{3} \beta_{2}\left(\alpha_{1}+\alpha_{2}\right) \int_{a}^{b} \frac{1}{q(z)} d z \\
& \left.+E_{4} \lambda_{3}\left(\alpha_{1}+\alpha_{2}\right) \int_{\xi}^{b} \int_{a}^{s} \frac{1}{p(z)} d z d s+R E_{3} \int_{a}^{t} \frac{1}{p(z)} d z\right)\left(\int_{a}^{\eta} \frac{\lambda_{2} \mu_{2}}{q(s)} \int_{a}^{s} g_{1}(z) d z d s\right) \\
& +\left(E_{2} \alpha_{2} \lambda_{3}(b-\xi) \int_{a}^{b} \frac{1}{p(z)} d z-E_{1} \lambda_{1} \lambda_{3}(b-\xi) \int_{a}^{\eta} \int_{a}^{s} \frac{1}{q(z)} d z d s\right. \\
& +E_{1} \beta_{2}\left(\alpha_{1}+\alpha_{2}\right) \int_{a}^{b} \frac{1}{q(z)} d z-E_{2} \lambda_{3}\left(\alpha_{1}+\alpha_{2}\right) \int_{\xi}^{b} \int_{a}^{s} \frac{1}{p(z)} d z d s \\
& \left.-R E_{1} \int_{a}^{t} \frac{1}{p(z)} d z\right)\left(\frac{\beta_{4} \mu_{2}}{q(b)} \int_{a}^{b} g_{1}(z) d z\right)+\left(-E_{2} \alpha_{2} \lambda_{3}(b-\xi) \int_{a}^{b} \frac{1}{p(z)} d z\right. \\
& +E_{1} \lambda_{1} \lambda_{3}(b-\xi) \int_{a}^{\eta} \int_{a}^{s} \frac{1}{q(z)} d z d s-E_{1} \beta_{2}\left(\alpha_{1}+\alpha_{2}\right) \int_{a}^{b} \frac{1}{q(z)} d z \\
& +E_{2} \lambda_{3}\left(\alpha_{1}+\alpha_{2}\right) \int_{\xi}^{b} \int_{a}^{s} \frac{1}{p(z)} d z d s \\
& \left.\left.+R E_{1} \int_{a}^{t} \frac{1}{p(z)} d z\right)\left(\int_{\xi}^{b} \frac{\lambda_{4} \mu_{1}}{p(s)} \int_{a}^{s} f_{1}(z) d z d s\right)\right]^{\prime}
\end{aligned}
$$

where

$$
\begin{aligned}
R & =\left(\alpha_{1}+\alpha_{2}\right)\left(\beta_{1}+\beta_{2}\right)-\lambda_{1} \lambda_{3}(\eta-a)(b-\xi) \\
E & =E_{1} E_{4}-E_{2} E_{3} \\
E_{1} & =\frac{\alpha_{3}}{p(a)}+\frac{\alpha_{4}}{p(b)}, E_{2}=\int_{a}^{\eta} \frac{\lambda_{2}}{q(s)} d s, E_{3}=\int_{\xi}^{b} \frac{\lambda_{4}}{p(s)} d s, E_{4}=\frac{\beta_{3}}{q(a)}+\frac{\beta_{4}}{q(b)}
\end{aligned}
$$

Proof. Firstly, we get the following formulas of solutions by integrating the linear differential equations (2.1) twice from $a$ to $t$ as

$$
u(t)=C_{1}+C_{2} \int_{a}^{t} \frac{1}{p(z)} d z+\int_{a}^{t}\left(\frac{\mu_{1}}{p(u)} \int_{a}^{u} f_{1}(z) d z\right) d u
$$

and

$$
v(t)=C_{3}+C_{4} \int_{a}^{t} \frac{1}{q(z)} d z+\int_{a}^{t}\left(\frac{\mu_{2}}{q(u)} \int_{a}^{u} g_{1}(z) d z\right) d u,
$$

where $C_{i}, i=1, \ldots, 4$, are arbitrary unknown real constants. By using the coupled boundary conditions given by (2.1) in (2.5) and (2.6), we obtain the following system:

$$
\begin{aligned}
\left(\alpha_{1}+\alpha_{2}\right) C_{1}-\lambda_{1}(\eta-a) C_{3}= & -\int_{a}^{b} \frac{\alpha_{2}}{p(z)} d z C_{2}+\int_{a}^{\eta} \int_{a}^{s}\left(\frac{\lambda_{1} \mu_{2}}{q(u)} \int_{a}^{u} g_{1}(z) d z\right) d u d s \\
& +\int_{a}^{\eta} \int_{a}^{s} \frac{\lambda_{1}}{q(z)} d z d s C_{4}-\int_{a}^{b}\left(\frac{\alpha_{2} \mu_{1}}{p(u)} \int_{a}^{u} f_{1}(z) d z\right) d u
\end{aligned}
$$




$$
\begin{aligned}
-\lambda_{3}(b-\xi) C_{1}+\left(\beta_{1}+\beta_{2}\right) C_{3}= & -\int_{a}^{b} \frac{\beta_{2}}{q(z)} d z C_{4}+\int_{\xi}^{b} \int_{a}^{s}\left(\frac{\lambda_{3} \mu_{1}}{p(u)} \int_{a}^{u} f_{1}(z) d z\right) d u d s \\
& +\int_{\xi}^{b} \int_{a}^{s} \frac{\lambda_{3}}{p(z)} d z d s C_{2}-\int_{a}^{b}\left(\frac{\beta_{2} \mu_{2}}{q(u)} \int_{a}^{u} g_{1}(z) d z\right) d u,
\end{aligned}
$$

$$
\left(\frac{\alpha_{3}}{p(a)}+\frac{\alpha_{4}}{p(b)}\right) C_{2}-\int_{a}^{\eta} \frac{\lambda_{2}}{q(s)} d s C_{4}=-\frac{\alpha_{4} \mu_{1}}{p(b)} \int_{a}^{b} f_{1}(z) d z+\int_{a}^{\eta} \frac{\lambda_{2} \mu_{2}}{q(s)} \int_{a}^{s} g_{1}(z) d z d s,
$$

$-\int_{\xi}^{b} \frac{\lambda_{4}}{p(s)} d s C_{2}+\left(\frac{\beta_{3}}{q(a)}+\frac{\beta_{4}}{q(b)}\right) C_{4}=-\frac{\beta_{4} \mu_{2}}{q(b)} \int_{a}^{b} g_{1}(z) d z+\int_{\xi}^{b} \frac{\lambda_{4} \mu_{1}}{p(s)} \int_{a}^{s} f_{1}(z) d z d s$.

Solving (2.9) and (2.10), we obtain that

$$
\begin{aligned}
C_{2}= & \frac{1}{E}\left[E_{4}\left(-\frac{\alpha_{4} \mu_{1}}{p(b)} \int_{a}^{b} f_{1}(z) d z+\int_{a}^{\eta} \frac{\lambda_{2} \mu_{2}}{q(s)} \int_{a}^{s} g_{1}(z) d z d s\right)\right. \\
& \left.+E_{2}\left(-\frac{\beta_{4} \mu_{2}}{q(b)} \int_{a}^{b} g_{1}(z) d z+\int_{\xi}^{b} \frac{\lambda_{4} \mu_{1}}{p(s)} \int_{a}^{s} f_{1}(z) d z d s\right)\right]
\end{aligned}
$$

and

$$
\begin{aligned}
C_{4}= & \frac{1}{E}\left[E_{3}\left(-\frac{\alpha_{4} \mu_{1}}{p(b)} \int_{a}^{b} f_{1}(z) d z+\int_{a}^{\eta} \frac{\lambda_{2} \mu_{2}}{q(s)} \int_{a}^{s} g_{1}(z) d z d s\right)\right. \\
& \left.+E_{1}\left(-\frac{\beta_{4} \mu_{2}}{q(b)} \int_{a}^{b} g_{1}(z) d z+\int_{\xi}^{b} \frac{\lambda_{4} \mu_{1}}{p(s)} \int_{a}^{s} f_{1}(z) d z d s\right)\right] .
\end{aligned}
$$

Using the values $C_{2}$ and $C_{4}$ in (2.7) and (2.8) then we find

$$
\begin{aligned}
C_{1}= & \frac{1}{R}\left[-\alpha_{2}\left(\beta_{1}+\beta_{2}\right) \int_{a}^{b}\left(\frac{\mu_{1}}{p(u)} \int_{a}^{u} f_{1}(z) d z\right) d u\right. \\
& +\lambda_{1}\left(\beta_{1}+\beta_{2}\right) \int_{a}^{\eta} \int_{a}^{s}\left(\frac{\mu_{2}}{q(u)} \int_{a}^{u} g_{1}(z) d z\right) d u d s-\lambda_{1} \beta_{2}(\eta-a) \int_{a}^{b}\left(\frac{\mu_{2}}{q(u)} \int_{a}^{u} g_{1}(z) d z\right) d u \\
& +\lambda_{1} \lambda_{3}(\eta-a) \int_{\xi}^{b} \int_{a}^{s}\left(\frac{\mu_{1}}{p(u)} \int_{a}^{u} f_{1}(z) d z\right) d u d s \\
& +\frac{1}{E}\left[\left(E_{4} \alpha_{2}\left(\beta_{1}+\beta_{2}\right) \int_{a}^{b} \frac{1}{p(z)} d z-E_{3} \lambda_{1}\left(\beta_{1}+\beta_{2}\right) \int_{a}^{\eta} \int_{a}^{s} \frac{1}{q(z)} d z d s\right.\right. \\
& \left.+E_{3} \lambda_{1} \beta_{2}(\eta-a) \int_{a}^{b} \frac{1}{q(z)} d z-E_{4} \lambda_{1} \lambda_{3}(\eta-a) \int_{\xi}^{b} \int_{a}^{s} \frac{1}{p(z)} d z d s\right)\left(\frac{\alpha_{4} \mu_{1}}{p(b)} \int_{a}^{b} f_{1}(z) d z\right) \\
& +\left(-E_{4} \alpha_{2}\left(\beta_{1}+\beta_{2}\right) \int_{a}^{b} \frac{1}{p(z)} d z+E_{3} \lambda_{1}\left(\beta_{1}+\beta_{2}\right) \int_{a}^{\eta} \int_{a}^{s} \frac{1}{q(z)} d z d s\right. \\
& \left.-E_{3} \lambda_{1} \beta_{2}(\eta-a) \int_{a}^{b} \frac{1}{q(z)} d z+E_{4} \lambda_{1} \lambda_{3}(\eta-a) \int_{\xi}^{b} \int_{a}^{s} \frac{1}{p(z)} d z d s\right)\left(\int_{a}^{\eta} \frac{\lambda_{2} \mu_{2}}{q(s)} \int_{a}^{s} g_{1}(z) d z d s\right) \\
& +\left(E_{2} \alpha_{2}\left(\beta_{1}+\beta_{2}\right) \int_{a}^{b} \frac{1}{p(z)} d z-E_{1} \lambda_{1}\left(\beta_{1}+\beta_{2}\right) \int_{a}^{\eta} \int_{a}^{s} \frac{1}{q(z)} d z d s\right.
\end{aligned}
$$


1484

$$
\begin{aligned}
& \left.+E_{1} \lambda_{1} \beta_{2}(\eta-a) \int_{a}^{b} \frac{1}{q(z)} d z-E_{2} \lambda_{1} \lambda_{3}(\eta-a) \int_{\xi}^{b} \int_{a}^{s} \frac{1}{p(z)} d z d s\right)\left(\frac{\beta_{4} \mu_{2}}{q(b)} \int_{a}^{b} g_{1}(z) d z\right) \\
& +\left(-E_{2} \alpha_{2}\left(\beta_{1}+\beta_{2}\right) \int_{a}^{b} \frac{1}{p(z)} d z+E_{1} \lambda_{1}\left(\beta_{1}+\beta_{2}\right) \int_{a}^{\eta} \int_{a}^{s} \frac{1}{q(z)} d z d s\right. \\
& -E_{1} \lambda_{1} \beta_{2}(\eta-a) \int_{a}^{b} \frac{1}{q(z)} d z \\
& \left.\left.\left.+E_{2} \lambda_{1} \lambda_{3}(\eta-a) \int_{\xi}^{b} \int_{a}^{s} \frac{1}{p(z)} d z d s\right)\left(\int_{\xi}^{b} \frac{\lambda_{4} \mu_{1}}{p(s)} \int_{a}^{s} f_{1}(z) d z d s\right)\right]\right]
\end{aligned}
$$

and

$$
\begin{aligned}
C_{3}= & \frac{1}{R}\left[-\alpha_{2} \lambda_{3}(b-\xi) \int_{a}^{b}\left(\frac{\mu_{1}}{p(u)} \int_{a}^{u} f_{1}(z) d z\right) d u\right. \\
& +\lambda_{1} \lambda_{3}(b-\xi) \int_{a}^{\eta} \int_{a}^{s}\left(\frac{\mu_{2}}{q(u)} \int_{a}^{u} g_{1}(z) d z\right) d u d s-\beta_{2}\left(\alpha_{1}+\alpha_{2}\right) \int_{a}^{b}\left(\frac{\mu_{2}}{q(u)} \int_{a}^{u} g_{1}(z) d z\right) d u \\
& \left.+\lambda_{3}\left(\alpha_{1}+\alpha_{2}\right) \int_{\xi}^{b} \int_{a}^{s}\left(\frac{\mu_{1}}{p(u)} \int_{a}^{u} f_{1}(z) d z\right) d u d s\right] \\
& +\frac{1}{E}\left[\left(E_{4} \alpha_{2} \lambda_{3}(b-\xi) \int_{a}^{b} \frac{1}{p(z)} d z-E_{3} \lambda_{1} \lambda_{3}(b-\xi) \int_{a}^{\eta} \int_{a}^{s} \frac{1}{q(z)} d z d s\right.\right. \\
& \left.+E_{3} \beta_{2}\left(\alpha_{1}+\alpha_{2}\right) \int_{a}^{b} \frac{1}{q(z)} d z-E_{4} \lambda_{3}\left(\alpha_{1}+\alpha_{2}\right) \int_{\xi}^{b} \int_{a}^{s} \frac{1}{p(z)} d z d s\right)\left(\frac{\alpha_{4} \mu_{1}}{p(b)} \int_{a}^{b} f_{1}(z) d z\right) \\
& +\left(-E_{4} \alpha_{2} \lambda_{3}(b-\xi) \int_{a}^{b} \frac{1}{p(z)} d z+E_{3} \lambda_{1} \lambda_{3}(b-\xi) \int_{a}^{\eta} \int_{a}^{s} \frac{1}{q(z)} d z d s\right. \\
& \left.-E_{3} \beta_{2}\left(\alpha_{1}+\alpha_{2}\right) \int_{a}^{b} \frac{1}{q(z)} d z+E_{4} \lambda_{3}\left(\alpha_{1}+\alpha_{2}\right) \int_{\xi}^{b} \int_{a}^{s} \frac{1}{p(z)} d z d s\right)\left(\int_{a}^{\eta} \frac{\lambda_{2} \mu_{2}}{q(s)} \int_{a}^{s} g_{1}(z) d z d s\right) \\
& +\left(E_{2} \alpha_{2} \lambda_{3}(b-\xi) \int_{a}^{b} \frac{1}{p(z)} d z-E_{1} \lambda_{1} \lambda_{3}(b-\xi) \int_{a}^{\eta} \int_{a}^{s} \frac{1}{q(z)} d z d s\right. \\
& \left.+E_{1} \beta_{2}\left(\alpha_{1}+\alpha_{2}\right) \int_{a}^{b} \frac{1}{q(z)} d z-E_{2} \lambda_{3}\left(\alpha_{1}+\alpha_{2}\right) \int_{\xi}^{b} \int_{a}^{s} \frac{1}{p(z)} d z d s\right)\left(\frac{\beta_{4} \mu_{2}}{q(b)} \int_{a}^{b} g_{1}(z) d z\right) \\
& \left.+\left(-E_{2} \lambda_{3}\left(\alpha_{1}+\alpha_{2}\right) \int_{\xi}^{b} \int_{a}^{s} \frac{1}{p(z)} d z d s\right)\left(\int_{\xi} \frac{\lambda_{4} \mu_{1}}{p(s)} \int_{a}^{s} f_{1}(z) d z d s\right)\right] . \\
& -E_{1} \beta_{2}\left(\alpha_{1}+\alpha_{2}\right) \int_{a}^{b} \frac{1}{q(z)} d z \\
& (2-\xi) \frac{1}{p(z)} d z+E_{1} \lambda_{1} \lambda_{3}(b-\xi) \int_{a}^{\eta} \int_{a}^{s} \frac{1}{q(z)} d z d s
\end{aligned}
$$

Substituting the values of $C_{1}, C_{2}$ and $C_{3}, C_{4}$ in (2.5) and (2.6) respectively, we have the solutions (2.2) and (2.3). By direct computation, we can obtain the converse of the lemma. This completes the proof. 


\section{MAIN RESULTS}

Let $(\mathcal{F},\|\cdot\|)$ denote the Banach space of all continuous real valued functions where $\mathcal{F}=\{u(t) \mid u(t) \in C([a, b], \mathbb{R})\}$ and $\|u\|=\sup \{|u(t)|, \quad t \in[a, b]\}$. Evidently the product space $(\mathcal{F} \times \mathcal{F},\|(u, v)\|)$ is a Banach space with the norm given by $\|(u, v)\|=$ $\|u\|+\|v\|$ for any $(u, v) \in \mathcal{F} \times \mathcal{F}$.

In view of Lemma 2.1, we transform the problem (1.1) into an equivalent fixed point problem as:

$$
(u, v)=\mathcal{H}(u, v)
$$

where $\mathcal{H}: \mathcal{F} \times \mathcal{F} \rightarrow \mathcal{F} \times \mathcal{F}$ is defined by

$$
\mathcal{H}(u, v)(t):=\left(\mathcal{H}_{1}(u, v)(t), \mathcal{H}_{2}(u, v)(t)\right)
$$

where

$$
\begin{aligned}
& \mathcal{H}_{1}(u, v)(t) \\
& =\int_{a}^{t}\left(\frac{\mu_{1}}{p(u)} \int_{a}^{u} f(z, u(z), v(z)) d z\right) d u \\
& +\frac{1}{R}\left[-\alpha_{2}\left(\beta_{1}+\beta_{2}\right) \int_{a}^{b}\left(\frac{\mu_{1}}{p(u)} \int_{a}^{u} f(z, u(z), v(z)) d z\right) d u\right. \\
& +\lambda_{1}\left(\beta_{1}+\beta_{2}\right) \int_{a}^{\eta} \int_{a}^{s}\left(\frac{\mu_{2}}{q(u)} \int_{a}^{u} g(z, u(z), v(z)) d z\right) d u d s \\
& -\lambda_{1} \beta_{2}(\eta-a) \int_{a}^{b}\left(\frac{\mu_{2}}{q(u)} \int_{a}^{u} g(z, u(z), v(z)) d z\right) d u \\
& \left.+\lambda_{1} \lambda_{3}(\eta-a) \int_{\xi}^{b} \int_{a}^{s}\left(\frac{\mu_{1}}{p(u)} \int_{a}^{u} f(z, u(z), v(z)) d z\right) d u d s\right] \\
& +\frac{1}{E R}\left[\left(E_{4} \alpha_{2}\left(\beta_{1}+\beta_{2}\right) \int_{a}^{b} \frac{1}{p(z)} d z-E_{3} \lambda_{1}\left(\beta_{1}+\beta_{2}\right) \int_{a}^{\eta} \int_{a}^{s} \frac{1}{q(z)} d z d s\right.\right. \\
& +E_{3} \lambda_{1} \beta_{2}(\eta-a) \int_{a}^{b} \frac{1}{q(z)} d z-E_{4} \lambda_{1} \lambda_{3}(\eta-a) \int_{\xi}^{b} \int_{a}^{s} \frac{1}{p(z)} d z d s \\
& \left.-R E_{4} \int_{a}^{t} \frac{1}{p(z)} d z\right)\left(\frac{\alpha_{4} \mu_{1}}{p(b)} \int_{a}^{b} f(z, u(z), v(z)) d z\right)+\left(-E_{4} \alpha_{2}\left(\beta_{1}+\beta_{2}\right) \int_{a}^{b} \frac{1}{p(z)} d z\right. \\
& +E_{3} \lambda_{1}\left(\beta_{1}+\beta_{2}\right) \int_{a}^{\eta} \int_{a}^{s} \frac{1}{q(z)} d z d s-E_{3} \lambda_{1} \beta_{2}(\eta-a) \int_{a}^{b} \frac{1}{q(z)} d z \\
& +E_{4} \lambda_{1} \lambda_{3}(\eta-a) \int_{\xi}^{b} \int_{a}^{s} \frac{1}{p(z)} d z d s \\
& \left.+R E_{4} \int_{a}^{t} \frac{1}{p(z)} d z\right)\left(\int_{a}^{\eta} \frac{\lambda_{2} \mu_{2}}{q(s)} \int_{a}^{s} g(z, u(z), v(z)) d z d s\right)
\end{aligned}
$$




$$
\begin{aligned}
+ & \left(E_{2} \alpha_{2}\left(\beta_{1}+\beta_{2}\right) \int_{a}^{b} \frac{1}{p(z)} d z-E_{1} \lambda_{1}\left(\beta_{1}+\beta_{2}\right) \int_{a}^{\eta} \int_{a}^{s} \frac{1}{q(z)} d z d s\right. \\
& +E_{1} \lambda_{1} \beta_{2}(\eta-a) \int_{a}^{b} \frac{1}{q(z)} d z-E_{2} \lambda_{1} \lambda_{3}(\eta-a) \int_{\xi}^{b} \int_{a}^{s} \frac{1}{p(z)} d z d s \\
& \left.-R E_{2} \int_{a}^{t} \frac{1}{p(z)} d z\right)\left(\frac{\beta_{4} \mu_{2}}{q(b)} \int_{a}^{b} g(z, u(z), v(z)) d z\right)+\left(-E_{2} \alpha_{2}\left(\beta_{1}+\beta_{2}\right) \int_{a}^{b} \frac{1}{p(z)} d z\right. \\
& +E_{1} \lambda_{1}\left(\beta_{1}+\beta_{2}\right) \int_{a}^{\eta} \int_{a}^{s} \frac{1}{q(z)} d z d s-E_{1} \lambda_{1} \beta_{2}(\eta-a) \int_{a}^{b} \frac{1}{q(z)} d z \\
& +E_{2} \lambda_{1} \lambda_{3}(\eta-a) \int_{\xi}^{b} \int_{a}^{s} \frac{1}{p(z)} d z d s \\
(3.3)+ & \left.\left.R E_{2} \int_{a}^{t} \frac{1}{p(z)} d z\right)\left(\int_{\xi}^{b} \frac{\lambda_{4} \mu_{1}}{p(s)} \int_{a}^{s} f(z, u(z), v(z)) d z d s\right)\right]
\end{aligned}
$$

and

$$
\begin{aligned}
& \mathcal{H}_{2}(u, v)(t) \\
& =\int_{a}^{t}\left(\frac{\mu_{2}}{q(u)} \int_{a}^{u} g(z, u(z), v(z)) d z\right) d u \\
& +\frac{1}{R}\left[-\alpha_{2} \lambda_{3}(b-\xi) \int_{a}^{b}\left(\frac{\mu_{1}}{p(u)} \int_{a}^{u} f(z, u(z), v(z)) d z\right) d u\right. \\
& +\lambda_{1} \lambda_{3}(b-\xi) \int_{a}^{\eta} \int_{a}^{s}\left(\frac{\mu_{2}}{q(u)} \int_{a}^{u} g(z, u(z), v(z)) d z\right) d u d s \\
& -\beta_{2}\left(\alpha_{1}+\alpha_{2}\right) \int_{a}^{b}\left(\frac{\mu_{2}}{q(u)} \int_{a}^{u} g(z, u(z), v(z)) d z\right) d u \\
& \left.+\lambda_{3}\left(\alpha_{1}+\alpha_{2}\right) \int_{\xi}^{b} \int_{a}^{s}\left(\frac{\mu_{1}}{p(u)} \int_{a}^{u} f(z, u(z), v(z)) d z\right) d u d s\right] \\
& +\frac{1}{E R}\left[\left(E_{4} \alpha_{2} \lambda_{3}(b-\xi) \int_{a}^{b} \frac{1}{p(z)} d z-E_{3} \lambda_{1} \lambda_{3}(b-\xi) \int_{a}^{\eta} \int_{a}^{s} \frac{1}{q(z)} d z d s\right.\right. \\
& +E_{3} \beta_{2}\left(\alpha_{1}+\alpha_{2}\right) \int_{a}^{b} \frac{1}{q(z)} d z-E_{4} \lambda_{3}\left(\alpha_{1}+\alpha_{2}\right) \int_{\xi}^{b} \int_{a}^{s} \frac{1}{p(z)} d z d s \\
& \left.-R E_{3} \int_{a}^{t} \frac{1}{p(z)} d z\right)\left(\frac{\alpha_{4} \mu_{1}}{p(b)} \int_{a}^{b} f(z, u(z), v(z)) d z\right)+\left(-E_{4} \alpha_{2} \lambda_{3}(b-\xi) \int_{a}^{b} \frac{1}{p(z)} d z\right. \\
& +E_{3} \lambda_{1} \lambda_{3}(b-\xi) \int_{a}^{\eta} \int_{a}^{s} \frac{1}{q(z)} d z d s-E_{3} \beta_{2}\left(\alpha_{1}+\alpha_{2}\right) \int_{a}^{b} \frac{1}{q(z)} d z \\
& +E_{4} \lambda_{3}\left(\alpha_{1}+\alpha_{2}\right) \int_{\xi}^{b} \int_{a}^{s} \frac{1}{p(z)} d z d s \\
& \left.+R E_{3} \int_{a}^{t} \frac{1}{p(z)} d z\right)\left(\int_{a}^{\eta} \frac{\lambda_{2} \mu_{2}}{q(s)} \int_{a}^{s} g(z, u(z), v(z)) d z d s\right)
\end{aligned}
$$




$$
\begin{aligned}
+ & \left(E_{2} \alpha_{2} \lambda_{3}(b-\xi) \int_{a}^{b} \frac{1}{p(z)} d z-E_{1} \lambda_{1} \lambda_{3}(b-\xi) \int_{a}^{\eta} \int_{a}^{s} \frac{1}{q(z)} d z d s\right. \\
& +E_{1} \beta_{2}\left(\alpha_{1}+\alpha_{2}\right) \int_{a}^{b} \frac{1}{q(z)} d z-E_{2} \lambda_{3}\left(\alpha_{1}+\alpha_{2}\right) \int_{\xi}^{b} \int_{a}^{s} \frac{1}{p(z)} d z d s \\
& \left.-R E_{1} \int_{a}^{t} \frac{1}{p(z)} d z\right)\left(\frac{\beta_{4} \mu_{2}}{q(b)} \int_{a}^{b} g(z, u(z), v(z)) d z\right)+\left(-E_{2} \alpha_{2} \lambda_{3}(b-\xi) \int_{a}^{b} \frac{1}{p(z)} d z\right. \\
& +E_{1} \lambda_{1} \lambda_{3}(b-\xi) \int_{a}^{\eta} \int_{a}^{s} \frac{1}{q(z)} d z d s-E_{1} \beta_{2}\left(\alpha_{1}+\alpha_{2}\right) \int_{a}^{b} \frac{1}{q(z)} d z \\
& +E_{2} \lambda_{3}\left(\alpha_{1}+\alpha_{2}\right) \int_{\xi}^{b} \int_{a}^{s} \frac{1}{p(z)} d z d s \\
(3.4)+ & \left.\left.R E_{1} \int_{a}^{t} \frac{1}{p(z)} d z\right)\left(\int_{\xi}^{b} \frac{\lambda_{4} \mu_{1}}{p(s)} \int_{a}^{s} f(z, u(z), v(z)) d z d s\right)\right]
\end{aligned}
$$

Note that the problem (1.1) has solutions if and only if the operator (3.2) has fixed points.

For the sake of computational convenience, we set:

$$
\mathcal{E}_{1}=\mathcal{D}_{1}+\mathcal{D}_{3}, \mathcal{E}_{2}=\mathcal{D}_{2}+\mathcal{D}_{4}
$$

and

$$
\mathcal{E}=\min \left\{1-\left(\mathcal{E}_{1} k_{1}+\mathcal{E}_{2} \hat{k}_{1}\right), 1-\left(\mathcal{E}_{1} k_{2}+\mathcal{E}_{2} \hat{k}_{2}\right)\right\}, k_{i}, \hat{k}_{i} \text { are given in }(S 1)
$$

where

$$
\begin{aligned}
\mathcal{D}_{1}= & \frac{\mu_{1}}{|R \bar{p}|}\left[\frac{(b-a)^{2}}{2}\left(|R|+\alpha_{2}\left(\beta_{1}+\beta_{2}\right)\right)+\frac{\lambda_{1} \lambda_{2}(\eta-a)\left[(b-a)^{3}-(\xi-a)^{3}\right]}{6}\right] \\
& +\frac{1}{|R E|}\left[\left(\frac{E_{4} \alpha_{2}\left(\beta_{1}+\beta_{2}\right)(b-a)}{\bar{p}}+\frac{E_{3} \lambda_{1}\left(\beta_{1}+\beta_{2}\right)(\eta-a)^{2}}{\bar{q} q}+\frac{E_{3} \lambda_{1} \beta_{2}(\eta-a)(b-a)}{\bar{q}}\right.\right. \\
& \left.+\frac{E_{4} \lambda_{1} \lambda_{3}(\eta-a)\left[(b-a)^{2}-(\xi-a)^{2}\right]}{2 \bar{p}}+\frac{R E_{4}(b-a)}{\bar{p}}\right)\left(\frac{\alpha_{4} \mu_{1}(b-a)}{|p(b)|}\right) \\
& +\left(\frac{E_{2} \alpha_{2}\left(\beta_{1}+\beta_{2}\right)(b-a)}{\bar{p}}+\frac{E_{1} \lambda_{1}\left(\beta_{1}+\beta_{2}\right)(\eta-a)^{2}}{2 \bar{q}}+\frac{E_{1} \lambda_{1} \beta_{2}(\eta-a)(b-a)}{\bar{q}}\right. \\
& \left.\left.+\frac{E_{2} \lambda_{1} \lambda_{3}(\eta-a)\left[(b-a)^{2}-(\xi-a)^{2}\right]}{2 \bar{p}}+\frac{R E_{2}(b-a)}{\bar{p}}\right)\left(\frac{\lambda_{4} \mu_{1}\left[(b-a)^{2}-(\xi-a)^{2}\right]}{2 \bar{p}}\right)\right], \\
\mathcal{D}_{2}= & \frac{\mu_{2}}{|2 R \bar{q}|}\left[\frac{\lambda_{1}\left(\beta_{1}+\beta_{2}\right)(\eta-a)^{3}}{3}+\lambda_{1} \beta_{2}(\eta-a)(b-a)^{2}\right] \\
& +\frac{1}{|R E|}\left[\left(\frac{E_{4} \alpha_{2}\left(\beta_{1}+\beta_{2}\right)(b-a)}{\bar{p}}+\frac{E_{3} \lambda_{1}\left(\beta_{1}+\beta_{2}\right)(\eta-a)^{2}}{2 \bar{q}}+\frac{E_{3} \lambda_{1} \beta_{2}(\eta-a)(b-a)}{\bar{q}}\right.\right. \\
& \left.+\frac{E_{4} \lambda_{1} \lambda_{3}(\eta-a)\left[(b-a)^{2}-(\xi-a)^{2}\right]}{2 \bar{p}}+\frac{R E_{4}(b-a)}{\bar{p}}\right)\left(\frac{\lambda_{2} \mu_{2}(\eta-a)^{2}}{2 \bar{q}}\right) \\
& +\left(\frac{E_{2} \alpha_{2}\left(\beta_{1}+\beta_{2}\right)(b-a)}{\bar{p}}+\frac{E_{1} \lambda_{1}\left(\beta_{1}+\beta_{2}\right)(\eta-a)^{2}}{2 q}+\frac{E_{1} \lambda_{1} \beta_{2}(\eta-a)(b-a)}{\bar{q}}\right.
\end{aligned}
$$




$$
\begin{aligned}
&\left.\left.+\frac{E_{2} \lambda_{1} \lambda_{3}(\eta-a)\left[(b-a)^{2}-(\xi-a)^{2}\right]}{2 \bar{p}}+\frac{R E_{2}(b-a)}{\bar{p}}\right)\left(\frac{\beta_{4} \mu_{2}(b-a)}{|q(b)|}\right)\right], \\
& \mathcal{D}_{3}= \frac{\mu_{1}}{|R \bar{p}|}\left[\frac{(b-a)^{2}}{2}\left(\alpha_{2} \lambda_{3}(b-\xi)\right)+\frac{\lambda_{3}\left(\alpha_{1}+\alpha_{2}\right)\left[(b-a)^{3}-(\xi-a)^{3}\right]}{6}\right] \\
&+\frac{1}{R E}\left[\left(\frac{E_{4} \alpha_{2} \lambda_{3}(b-\xi)(b-a)}{\bar{p}}+\frac{E_{3} \lambda_{1} \lambda_{3}(b-\xi)(\eta-a)^{2}}{2 \bar{q}}+\frac{E_{3} \beta_{2}\left(\alpha_{1}+\alpha_{2}\right)(b-a)}{\bar{q}}\right.\right. \\
&\left.+\frac{E_{4} \lambda_{3}\left(\alpha_{1}+\alpha_{2}\right)\left[(b-a)^{2}-(\xi-a)^{2}\right]}{2 \bar{p}}+\frac{R E_{3}(b-a)}{\bar{p}}\right)\left(\frac{\alpha_{4} \mu_{1}(b-a)}{|p(b)|}\right) \\
&+\left(\frac{E_{2} \alpha_{2} \lambda_{3}(b-\xi)(b-a)}{\bar{p}}+\frac{E_{1} \lambda_{1} \lambda_{3}(b-\xi)(\eta-a)^{2}}{2 \bar{q}}+\frac{E_{1} \beta_{2}\left(\alpha_{1}+\alpha_{2}\right)(b-a)}{\bar{q}}\right) \\
&\left.\left.+\frac{E_{2} \lambda_{3}\left(\alpha_{1}+\alpha_{2}\right)\left[(b-a)^{2}-(\xi-a)^{2}\right]}{2 \bar{p}}+\frac{R E_{1}(b-a)}{\bar{p}}\right)\left(\frac{\lambda_{4} \mu_{1}\left[(b-a)^{2}-(\xi-a)^{2}\right]}{2 \bar{p}}\right)\right], \\
& \mathcal{D}_{4}= \frac{\mu_{2}}{|R \bar{q}|}\left[\frac{(b-a)^{2}}{2}\left(|R|+\beta_{2}\left(\alpha_{1}+\alpha_{2}\right)\right)+\frac{\lambda_{1} \lambda_{3}(b-\xi)(\eta-a)^{3}}{6}\right] \\
&+\frac{1}{R E}\left[\left(\frac{E_{4} \alpha_{2} \lambda_{3}(b-\xi)(b-a)}{\bar{p}}+\frac{E_{3} \lambda_{1} \lambda_{3}(b-\xi)(\eta-a)^{2}}{2 \bar{q}}+\frac{E_{3} \beta_{2}\left(\alpha_{1}+\alpha_{2}\right)(b-a)}{\bar{q}}\right.\right. \\
&\left.+\frac{E_{4} \lambda_{3}\left(\alpha_{1}+\alpha_{2}\right)\left[(b-a)^{2}-(\xi-a)^{2}\right]}{2 \bar{p}}+\frac{R E_{3}(b-a)}{\bar{p}}\right)\left(\frac{\lambda_{2} \mu_{2}(\eta-a)^{2}}{2 \bar{q}}\right) \\
&+\left(\frac{E_{2} \alpha_{2} \lambda_{3}(b-\xi)(b-a)}{\bar{p}}+\frac{E_{1} \lambda_{1} \lambda_{3}(b-\xi)(\eta-a)^{2}}{2 \bar{q}}+\frac{E_{1} \beta_{2}\left(\alpha_{1}+\alpha_{2}\right)(b-a)}{\bar{q}}\right. \\
&(3.8) \quad \bar{p}=\inf _{z \in[a, b]}|p(z)|, \bar{q}=\inf _{2 \in[a, b]}|q(z)| . \\
&(3.7)\left.\frac{R E_{1}(b-a)}{\bar{p} \lambda_{3}\left(\alpha_{1}+\alpha_{2}\right)\left[(b-a)^{2}-(\xi-a)^{2}\right]}\right), \\
& \beta_{4}(b-a) \\
&|q(b)|
\end{aligned}
$$

3.1. Existence results. In this section, we prove two existence results for the problem (1.1). The first is based on the Leray-Schauder alternative [10].

Lemma 3.1. (Leray-Schauder alternative). Let $\Omega$ be a Banach space, and $Y: \Omega \rightarrow \Omega$ be a completely continuous operator (i.e., a map restricted to any bounded set in $\Omega$ is compact). Let $\Gamma(Y)=\{y \in \Omega: y=\delta Y(y)$ for some $0<\delta<1\}$. Then either the set $\Gamma(Y)$ is unbounded or $Y$ has at least one fixed point.

Theorem 3.2. Assume that:

$\left(S_{1}\right)$ There exist real constants $k_{i}, \hat{k}_{i} \geq 0(i=1,2)$, and $k_{0}>0, \hat{k}_{0}>0$, such that $\forall u, v \in \mathbb{R}$, we have

$$
|f(t, u, v)| \leq k_{0}+k_{1}|u|+k_{2}|v|, \text { and }|g(t, u, v)| \leq \hat{k}_{0}+\hat{k}_{1}|u|+\hat{k}_{2}|v| .
$$

Then there exist at least one solution for the problem (1.1) on $[a, b]$, provided that

$$
\mathcal{E}_{1} k_{1}+\mathcal{E}_{2} \hat{k}_{1}<1 \text { and } \mathcal{E}_{1} k_{2}+\mathcal{E}_{2} \hat{k}_{2}<1
$$

where $\mathcal{E}_{i}(i=1,2)$ are given by $(3.5)$. 
Proof. The proof consists of two steps:

Step 1: We show that the operator $\mathcal{H}: \mathcal{F} \times \mathcal{F} \rightarrow \mathcal{F} \times \mathcal{F}$ defined by (3.2) is completely continuous. Since the functions $f$ and $g$ are continuous, it follows that the operators $\mathcal{H}_{1}$ and $\mathcal{H}_{2}$ are continuous. Consequently the operator $\mathcal{H}$ is continuous. Let $\rho \subset \mathcal{F} \times \mathcal{F}$ be a bounded set. Then, there exist positive constants $\mathcal{O}_{f}$ and $\mathcal{O}_{g}$ such that

$$
|f(t, u(t), v(t))| \leq \mathcal{O}_{f} \text {, and }|g(t, u(t), v(t))| \leq \mathcal{O}_{g}, \forall(u, v) \in \rho .
$$

Then, for any $(u, v) \in \rho$, we have

$$
\begin{aligned}
& \left|\mathcal{H}_{1}(u, v)(t)\right| \\
& \leq \int_{a}^{t}\left(\frac{\mu_{1}}{|p(u)|} \int_{a}^{u}|f(z, u(z), v(z))| d z\right) d u \\
& +\frac{1}{R}\left[\alpha_{2}\left(\beta_{1}+\beta_{2}\right) \int_{a}^{b}\left(\frac{\mu_{1}}{|p(u)|} \int_{a}^{u}|f(z, u(z), v(z))| d z\right) d u\right. \\
& +\lambda_{1}\left(\beta_{1}+\beta_{2}\right) \int_{a}^{\eta} \int_{a}^{s}\left(\frac{\mu_{2}}{|q(u)|} \int_{a}^{u}|g(z, u(z), v(z))| d z\right) d u d s \\
& +\lambda_{1} \beta_{2}(\eta-a) \int_{a}^{b}\left(\frac{\mu_{2}}{|q(u)|} \int_{a}^{u}|g(z, u(z), v(z))| d z\right) d u \\
& \left.+\lambda_{1} \lambda_{3}(\eta-a) \int_{\xi}^{b} \int_{a}^{s}\left(\frac{\mu_{1}}{|p(u)|} \int_{a}^{u}|f(z, u(z), v(z))| d z\right) d u d s\right] \\
& +\frac{1}{E R}\left[\left(E_{4} \alpha_{2}\left(\beta_{1}+\beta_{2}\right) \int_{a}^{b} \frac{1}{|p(z)|} d z+E_{3} \lambda_{1}\left(\beta_{1}+\beta_{2}\right) \int_{a}^{\eta} \int_{a}^{s} \frac{1}{|q(z)|} d z d s\right.\right. \\
& +E_{3} \lambda_{1} \beta_{2}(\eta-a) \int_{a}^{b} \frac{1}{|q(z)|} d z+E_{4} \lambda_{1} \lambda_{3}(\eta-a) \int_{\xi}^{b} \int_{a}^{s} \frac{1}{|p(z)|} d z d s \\
& \left.+R E_{4} \int_{a}^{t} \frac{1}{|p(z)|} d z\right)\left(\frac{\alpha_{4} \mu_{1}}{|p(b)|} \int_{a}^{b}|f(z, u(z), v(z))| d z\right)+\left(E_{4} \alpha_{2}\left(\beta_{1}+\beta_{2}\right) \int_{a}^{b} \frac{1}{|p(z)|} d z\right. \\
& +E_{3} \lambda_{1}\left(\beta_{1}+\beta_{2}\right) \int_{a}^{\eta} \int_{a}^{s} \frac{1}{|q(z)|} d z d s+E_{3} \lambda_{1} \beta_{2}(\eta-a) \int_{a}^{b} \frac{1}{|q(z)|} d z \\
& \left.+E_{4} \lambda_{1} \lambda_{3}(\eta-a) \int_{\xi}^{b} \int_{a}^{s} \frac{1}{|p(z)|} d z d s+R E_{4} \int_{a}^{t} \frac{1}{|p(z)|} d z\right) \\
& \times\left(\int_{a}^{\eta} \frac{\lambda_{2} \mu_{2}}{|q(s)|} \int_{a}^{s}|g(z, u(z), v(z))| d z d s\right)+\left(E_{2} \alpha_{2}\left(\beta_{1}+\beta_{2}\right) \int_{a}^{b} \frac{1}{|p(z)|} d z\right. \\
& +E_{1} \lambda_{1}\left(\beta_{1}+\beta_{2}\right) \int_{a}^{\eta} \int_{a}^{s} \frac{1}{|q(z)|} d z d s+E_{1} \lambda_{1} \beta_{2}(\eta-a) \int_{a}^{b} \frac{1}{|q(z)|} d z \\
& \left.+E_{2} \lambda_{1} \lambda_{3}(\eta-a) \int_{\xi}^{b} \int_{a}^{s} \frac{1}{|p(z)|} d z d s+R E_{2} \int_{a}^{t} \frac{1}{|p(z)|} d z\right)\left(\frac{\beta_{4} \mu_{2}}{|q(b)|} \int_{a}^{b}|g(z, u(z), v(z))| d z\right) \\
& +\left(E_{2} \alpha_{2}\left(\beta_{1}+\beta_{2}\right) \int_{a}^{b} \frac{1}{|p(z)|} d z+E_{1} \lambda_{1}\left(\beta_{1}+\beta_{2}\right) \int_{a}^{\eta} \int_{a}^{s} \frac{1}{|q(z)|} d z d s\right. \\
& +E_{1} \lambda_{1} \beta_{2}(\eta-a) \int_{a}^{b} \frac{1}{|q(z)|} d z+E_{2} \lambda_{1} \lambda_{3}(\eta-a) \int_{\xi}^{b} \int_{a}^{s} \frac{1}{|p(z)|} d z d s
\end{aligned}
$$




$$
\begin{aligned}
+ & \left.\left.R E_{2} \int_{a}^{t} \frac{1}{|p(z)|} d z\right)\left(\int_{\xi}^{b} \frac{\lambda_{4} \mu_{1}}{|p(s)|} \int_{a}^{s}|f(z, u(z), v(z))| d z d s\right)\right] \\
\leq & \mathcal{O}_{f}\left\{\frac{\mu_{1}}{|R \bar{p}|}\left[\frac{(b-a)^{2}}{2}\left(|R|+\alpha_{2}\left(\beta_{1}+\beta_{2}\right)\right)+\frac{\lambda_{1} \lambda_{2}(\eta-a)\left[(b-a)^{3}-(\xi-a)^{3}\right]}{6}\right]\right. \\
& +\frac{1}{|R E|}\left[\left(\frac{E_{4} \alpha_{2}\left(\beta_{1}+\beta_{2}\right)(b-a)}{\bar{p}}+\frac{E_{3} \lambda_{1}\left(\beta_{1}+\beta_{2}\right)(\eta-a)^{2}}{2 q}+\frac{E_{3} \lambda_{1} \beta_{2}(\eta-a)(b-a)}{\bar{q}}\right.\right. \\
& \left.+\frac{E_{4} \lambda_{1} \lambda_{3}(\eta-a)\left[(b-a)^{2}-(\xi-a)^{2}\right]}{2 \bar{p}}+\frac{R E_{4}(b-a)}{\bar{p}}\right)\left(\frac{\alpha_{4} \mu_{1}(b-a)}{|p(b)|}\right) \\
& +\left(\frac{E_{2} \alpha_{2}\left(\beta_{1}+\beta_{2}\right)(b-a)}{\bar{p}}+\frac{E_{1} \lambda_{1}\left(\beta_{1}+\beta_{2}\right)(\eta-a)^{2}}{2 \bar{q}}+\frac{E_{1} \lambda_{1} \beta_{2}(\eta-a)(b-a)}{\bar{q}}\right. \\
& \left.\left.\left.+\frac{E_{2} \lambda_{1} \lambda_{3}(\eta-a)\left[(b-a)^{2}-(\xi-a)^{2}\right]}{2 \bar{p}}+\frac{R E_{2}(b-a)}{\bar{p}}\right)\left(\frac{\lambda_{4} \mu_{1}\left[(b-a)^{2}-(\xi-a)^{2}\right]}{2 \bar{p}}\right)\right]\right\} \\
& +\mathcal{O}_{g}\left\{\frac{\mu_{2}}{|2 R \bar{q}|}\left[\frac{\lambda_{1}\left(\beta_{1}+\beta_{2}\right)(\eta-a)^{3}}{3}+\lambda_{1} \beta_{2}(\eta-a)(b-a)^{2}\right]\right. \\
+ & \left.\frac{1}{|R E|}\left[\left(\frac{E_{4} \alpha_{2}\left(\beta_{1}+\beta_{2}\right)(b-a)}{\bar{p}}+\frac{E_{3} \lambda_{1}\left(\beta_{1}+\beta_{2}\right)(\eta-a)^{2}}{2 \bar{q}}+\frac{E_{3} \lambda_{1} \beta_{2}(\eta-a)(b-a)}{\bar{q}}\right)\left(\frac{\beta_{4} \mu_{2}(b-a)}{|q(b)|}\right)\right]\right\}, \\
+ & \left.\frac{E_{4} \lambda_{1} \lambda_{3}(\eta-a)\left[(b-a)^{2}-(\xi-a)^{2}\right]}{2 \bar{p}}+\frac{R E_{4}(b-a)}{\bar{p}}\right)\left(\frac{\lambda_{2} \mu_{2}(\eta-a)^{2}}{2 \bar{q}}\right) \\
+ & \frac{E_{2} \alpha_{2}\left(\beta_{1}+\beta_{2}\right)(b-a)}{\bar{p}}+\frac{E_{1} \lambda_{1}\left(\beta_{1}+\beta_{2}\right)(\eta-a)^{2}}{2 q}+\frac{E_{1} \lambda_{1} \beta_{2}(\eta-a)(b-a)}{\bar{q} \lambda_{1} \lambda_{3}(\eta-a)\left[(b-a)^{2}-(\xi-a)^{2}\right]}
\end{aligned}
$$

which, on taking the norm for $t \in[a, b]$, yields $\left\|\mathcal{H}_{1}(u, v)\right\| \leq \mathcal{O}_{f} \mathcal{D}_{1}+\mathcal{O}_{g} \mathcal{D}_{2}$. Similarly, we have $\left\|\mathcal{H}_{2}(u, v)\right\| \leq \mathcal{O}_{f} \mathcal{D}_{3}+\mathcal{O}_{g} \mathcal{D}_{4}$, where $\mathcal{D}_{i}(i=1, \ldots, 4)$ are given by (3.7). From the above inequalities, it follows that the operator $\mathcal{H}$ is uniformly bounded, since

$$
\|\mathcal{H}(u, v)\| \leq \mathcal{O}_{f} \mathcal{E}_{1}+\mathcal{O}_{g} \mathcal{E}_{2}
$$

where $\mathcal{E}_{i}(i=1,2)$ are given by $(3.5)$.

Next, we show that $\mathcal{H}$ is an equicontinuous operator. Let $t_{1}, t_{2} \in[a, b]$ with $t_{1}<t_{2}$, this yields

$$
\begin{aligned}
\mid & \left|\mathcal{H}_{1}(u, v)\left(t_{2}\right)-\mathcal{H}_{1}(u, v)\left(t_{1}\right)\right| \\
= & \left.\mid \int_{a}^{t_{2}}\left(\frac{\mu_{1}}{p(u)} \int_{a}^{u} f(z, u(z), v(z)) d z\right) d u-\int_{a}^{t_{1}}\left(\frac{\mu_{1}}{p(u)} \int_{a}^{u} f(z, u(z), v(z))\right) d z\right) d u \\
& +\left(\frac{E_{4}}{E}\left(\int_{a}^{t_{2}} \frac{1}{p(z)} d z-\int_{a}^{t_{1}} \frac{1}{p(z)} d z\right)\left(\frac{\alpha_{4} \mu_{1}}{p(b)} \int_{a}^{b} f(z, u(z), v(z)) d z\right)\right) \\
& +\left(\frac{E_{4}}{E}\left(\int_{a}^{t_{2}} \frac{1}{p(z)} d z-\int_{a}^{t_{1}} \frac{1}{p(z)} d z\right)\left(\int_{a}^{\eta} \frac{\lambda_{2} \mu_{2}}{q(s)} \int_{a}^{s} g(z, u(z), v(z)) d z d s\right)\right) \\
& \left.+\left(\frac{E_{2}}{E} \int_{a}^{t_{2}} \frac{1}{p(z)} d z-\int_{a}^{t_{1}} \frac{1}{p(z)} d z\right)\left(\frac{\beta_{4} \mu_{2}}{q(b)} \int_{a}^{b} g(z, u(z), v(z)) d z\right)\right)
\end{aligned}
$$




$$
\begin{aligned}
& +\left(\frac{E_{2}}{E}\left(\int_{a}^{t_{2}} \frac{1}{p(z)} d z-\int_{a}^{t_{1}} \frac{1}{p(z)} d z\right)\left(\int_{\xi}^{b} \frac{\lambda_{4} \mu_{1}}{p(s)} \int_{a}^{s} f(z, u(z), v(z)) d z d s\right)\right) \mid \\
\leq & \mathcal{O}_{f}\left[\left(\frac{\mu_{1}}{|\bar{p}|}\right) \frac{\left(t_{2}-a\right)^{2}-\left(t_{1}-a\right)^{2}}{2}+\frac{E_{4}}{E|\bar{p}|}\left(\frac{\alpha_{4} \mu_{1}}{|p(b)|}\right)\left(t_{2}-t_{1}\right)(b-a)\right. \\
& \left.+\frac{E_{2}}{E|\bar{p}|} \frac{\left(\lambda_{4} \mu_{1}\right)\left(t_{2}-t_{1}\right)\left[(b-a)^{2}-(\xi-a)^{2}\right]}{2}\right] \\
& +\mathcal{O}_{g}\left[\frac{E_{4}}{E|\bar{p}|} \frac{\left(\lambda_{2} \mu_{2}\right)\left(t_{2}-t_{1}\right)(\eta-a)^{2}}{2 \bar{q}}+\frac{E_{2}}{E|\bar{p}|}\left(\frac{\beta_{4} \mu_{2}}{|q(b)|}\right)\left(t_{2}-t_{1}\right)(b-a)\right] \rightarrow 0 \\
& \text { as } t_{2} \rightarrow t_{1} \text { independent of }(u, v) .
\end{aligned}
$$

Similarly, we can find that

$$
\left|\mathcal{H}_{2}(u, v)\left(t_{2}\right)-\mathcal{H}_{2}(u, v)\left(t_{1}\right)\right| \rightarrow 0 \text { as } t_{2} \rightarrow t_{1} \text { independent of }(u, v) .
$$

Therefore, the operator $\mathcal{H}$ is equicontinuous.

Step 2: We verify that the set $\Phi=\{(u, v) \in \mathcal{F} \times \mathcal{F} \mid(u, v)=\delta \mathcal{H}(u, v), 0<\delta<1\}$ is bounded. Let $(u, v) \in \Phi$. Then $(u, v)=\delta \mathcal{H}(u, v)$, we have for any $t \in[a, b]$,

$$
u(t)=\delta \mathcal{H}_{1}(u, v)(t), v(t)=\delta \mathcal{H}_{2}(u, v)(t)
$$

Then, using the conditions $\left(S_{1}\right)$, we have

$$
\begin{aligned}
|u(t)| & \leq \mathcal{D}_{1}\left(k_{0}+k_{1}|u|+k_{2}|v|\right)+\mathcal{D}_{2}\left(\hat{k}_{0}+\hat{k}_{1}|u|+\hat{k}_{2}|v|\right) \\
& \leq \mathcal{D}_{1} k_{0}+\mathcal{D}_{2} \hat{k}_{0}+\left(\mathcal{D}_{1} k_{1}+\mathcal{D}_{2} \hat{k}_{1}\right)\|u\|+\left(\mathcal{D}_{1} k_{2}+\mathcal{D}_{2} \hat{k}_{2}\right)\|v\|,
\end{aligned}
$$

and

$$
\begin{aligned}
|v(t)| & \leq \mathcal{D}_{3}\left(k_{0}+k_{1}|u|+k_{2}|v|\right)+\mathcal{D}_{4}\left(\hat{k}_{0}+\hat{k}_{1}|u|+\hat{k}_{2}|v|\right) \\
& \leq \mathcal{D}_{3} k_{0}+\mathcal{D}_{4} \hat{k}_{0}+\left(\mathcal{D}_{3} k_{1}+\mathcal{D}_{4} \hat{k}_{1}\right)\|u\|+\left(\mathcal{D}_{3} k_{2}+\mathcal{D}_{4} \hat{k}_{2}\right)\|v\|
\end{aligned}
$$

which implies

$$
\begin{aligned}
\|u\|+\|v\| \leq & \left(\mathcal{D}_{1}+\mathcal{D}_{3}\right) k_{0}+\left(\mathcal{D}_{2}+\mathcal{D}_{4}\right) \hat{k}_{0}+\left[\left(\mathcal{D}_{1}+\mathcal{D}_{3}\right) k_{1}+\left(\mathcal{D}_{2}+\mathcal{D}_{4}\right) \hat{k}_{1}\right]\|u\| \\
& +\left[\left(\mathcal{D}_{1}+\mathcal{D}_{3}\right) k_{2}+\left(\mathcal{D}_{2}+\mathcal{D}_{4}\right) \hat{k}_{2}\right]\|v\| .
\end{aligned}
$$

By (3.6) and (3.9), we have

$$
\|(u, v)\| \leq \frac{\mathcal{E}_{1} k_{0}+\mathcal{E}_{2} \hat{k}_{0}}{\mathcal{E}} .
$$

This proves that set $\Phi$ is bounded and hence the Leray-Schauder alternative implies the operator $\mathcal{H}$ has at least one fixed point. Therefore, the problem (1.1) has at least one solution on $[a, b]$. This completes the proof.

Now, we discuss the second existence result for the problem (1.1) by applying the Schauder fixed point theorem [10]. 
Theorem 3.3. Assume that:

$\left(S_{2}\right)$ There exist nonnegative functions $\psi, \phi \in L(a, b)$ such that

$|f(t, u, v)| \leq \psi(t)+\epsilon_{1}|u|^{m_{1}}+\epsilon_{2}|v|^{m_{2}}, u, v \in \mathbb{R}, \epsilon_{1}, \epsilon_{2}>0,0<m_{1}, m_{2}<1$,

and

$|g(t, u, v)| \leq \phi(t)+\sigma_{1}|u|^{n_{1}}+\sigma_{2}|v|^{n_{2}}, u, v \in \mathbb{R}, \sigma_{1}, \sigma_{2}>0,0<n_{1}, n_{2}<1$.

Then there exists at least one solution for the problem (1.1) on $[a, b]$.

Proof. Let us fix

$$
\varphi \geqslant \max \left\{6 \mathcal{E}_{1}\|\psi\|, 6 \mathcal{E}_{2}\|\phi\|,\left(6 \epsilon_{1} \mathcal{E}_{1}\right)^{\frac{1}{1-m_{1}}},\left(6 \epsilon_{2} \mathcal{E}_{1}\right)^{\frac{1}{1-m_{2}}},\left(6 \sigma_{1} \mathcal{E}_{2}\right)^{\frac{1}{1-n_{1}}},\left(6 \sigma_{2} \mathcal{E}_{2}\right)^{\frac{1}{1-n_{2}}}\right\}
$$

We introduce a set $\Theta$ in a Banach space define by $\Theta=\{(u, v) \in \mathcal{F} \times \mathcal{F}:\|(u, v)\| \leq$ $\varphi\}$, and the operator $\mathcal{H}: \Theta \rightarrow \Theta$. For any $(u, v) \in \Theta$, we have

$$
\begin{aligned}
& \left|\mathcal{H}_{1}(u, v)(t)\right| \\
& \leq \int_{a}^{t}\left(\frac{\mu_{1}}{|p(u)|} \int_{a}^{u}|f(z, u(z), v(z))| d z\right) d u \\
& +\frac{1}{R}\left[\alpha_{2}\left(\beta_{1}+\beta_{2}\right) \int_{a}^{b}\left(\frac{\mu_{1}}{|p(u)|} \int_{a}^{u}|f(z, u(z), v(z))| d z\right) d u\right. \\
& +\lambda_{1}\left(\beta_{1}+\beta_{2}\right) \int_{a}^{\eta} \int_{a}^{s}\left(\frac{\mu_{2}}{|q(u)|} \int_{a}^{u}|g(z, u(z), v(z))| d z\right) d u d s \\
& +\lambda_{1} \beta_{2}(\eta-a) \int_{a}^{b}\left(\frac{\mu_{2}}{|q(u)|} \int_{a}^{u}|g(z, u(z), v(z))| d z\right) d u \\
& \left.+\lambda_{1} \lambda_{3}(\eta-a) \int_{\xi}^{b} \int_{a}^{s}\left(\frac{\mu_{1}}{|p(u)|} \int_{a}^{u}|f(z, u(z), v(z))| d z\right) d u d s\right] \\
& +\frac{1}{E R}\left[\left(E_{4} \alpha_{2}\left(\beta_{1}+\beta_{2}\right) \int_{a}^{b} \frac{1}{|p(z)|} d z+E_{3} \lambda_{1}\left(\beta_{1}+\beta_{2}\right) \int_{a}^{\eta} \int_{a}^{s} \frac{1}{|q(z)|} d z d s\right.\right. \\
& +E_{3} \lambda_{1} \beta_{2}(\eta-a) \int_{a}^{b} \frac{1}{|q(z)|} d z+E_{4} \lambda_{1} \lambda_{3}(\eta-a) \int_{\xi}^{b} \int_{a}^{s} \frac{1}{|p(z)|} d z d s \\
& \left.+R E_{4} \int_{a}^{t} \frac{1}{|p(z)|} d z\right)\left(\frac{\alpha_{4} \mu_{1}}{|p(b)|} \int_{a}^{b}|f(z, u(z), v(z))| d z\right)+\left(E_{4} \alpha_{2}\left(\beta_{1}+\beta_{2}\right) \int_{a}^{b} \frac{1}{|p(z)|} d z\right. \\
& +E_{3} \lambda_{1}\left(\beta_{1}+\beta_{2}\right) \int_{a}^{\eta} \int_{a}^{s} \frac{1}{|q(z)|} d z d s+E_{3} \lambda_{1} \beta_{2}(\eta-a) \int_{a}^{b} \frac{1}{|q(z)|} d z \\
& \left.+E_{4} \lambda_{1} \lambda_{3}(\eta-a) \int_{\xi}^{b} \int_{a}^{s} \frac{1}{|p(z)|} d z d s+R E_{4} \int_{a}^{t} \frac{1}{|p(z)|} d z\right) \\
& \times\left(\int_{a}^{\eta} \frac{\lambda_{2} \mu_{2}}{|q(s)|} \int_{a}^{s}|g(z, u(z), v(z))| d z d s\right)+\left(E_{2} \alpha_{2}\left(\beta_{1}+\beta_{2}\right) \int_{a}^{b} \frac{1}{|p(z)|} d z\right. \\
& +E_{1} \lambda_{1}\left(\beta_{1}+\beta_{2}\right) \int_{a}^{\eta} \int_{a}^{s} \frac{1}{|q(z)|} d z d s+E_{1} \lambda_{1} \beta_{2}(\eta-a) \int_{a}^{b} \frac{1}{|q(z)|} d z
\end{aligned}
$$




$$
\begin{aligned}
& \left.+E_{2} \lambda_{1} \lambda_{3}(\eta-a) \int_{\xi}^{b} \int_{a}^{s} \frac{1}{|p(z)|} d z d s+R E_{2} \int_{a}^{t} \frac{1}{|p(z)|} d z\right)\left(\frac{\beta_{4} \mu_{2}}{|q(b)|} \int_{a}^{b}|g(z, u(z), v(z))| d z\right) \\
& +\left(E_{2} \alpha_{2}\left(\beta_{1}+\beta_{2}\right) \int_{a}^{b} \frac{1}{|p(z)|} d z+E_{1} \lambda_{1}\left(\beta_{1}+\beta_{2}\right) \int_{a}^{\eta} \int_{a}^{s} \frac{1}{|q(z)|} d z d s\right. \\
& +E_{1} \lambda_{1} \beta_{2}(\eta-a) \int_{a}^{b} \frac{1}{|q(z)|} d z+E_{2} \lambda_{1} \lambda_{3}(\eta-a) \int_{\xi}^{b} \int_{a}^{s} \frac{1}{|p(z)|} d z d s \\
& \left.\left.+R E_{2} \int_{a}^{t} \frac{1}{|p(z)|} d z\right)\left(\int_{\xi}^{b} \frac{\lambda_{4} \mu_{1}}{|p(s)|} \int_{a}^{s}|f(z, u(z), v(z))| d z d s\right)\right] \\
& \leq\left(\psi(t)+\epsilon_{1}|u|^{m_{1}}+\epsilon_{2}|v|^{m_{2}}\right)\left\{\frac { 1 } { R } \left[\frac { \mu _ { 1 } } { | \overline { p } | } \left(R \frac{(b-a)^{2}}{2}+\frac{\alpha_{2}\left(\beta_{1}+\beta_{2}\right)(b-a)^{2}}{2}\right.\right.\right. \\
& \left.+\frac{\lambda_{1} \lambda_{2}(\eta-a)\left[(b-a)^{3}-(\xi-a)^{3}\right]}{6}\right) \\
& +\frac{1}{E}\left(\left(\frac{E_{4} \alpha_{2}\left(\beta_{1}+\beta_{2}\right)(b-a)}{\bar{p}}+\frac{E_{3} \lambda_{1}\left(\beta_{1}+\beta_{2}\right)(\eta-a)^{2}}{\overline{2 q}}+\frac{E_{3} \lambda_{1} \beta_{2}(\eta-a)(b-a)}{\bar{q}}\right.\right. \\
& \left.+\frac{E_{4} \lambda_{1} \lambda_{3}(\eta-a)\left[(b-a)^{2}-(\xi-a)^{2}\right]}{2 \bar{p}}+\frac{R E_{4}(b-a)}{\bar{p}}\right)\left(\frac{\alpha_{4} \mu_{1}(b-a)}{|p(b)|}\right) \\
& +\left(\frac{E_{2} \alpha_{2}\left(\beta_{1}+\beta_{2}\right)(b-a)}{\bar{p}}+\frac{E_{1} \lambda_{1}\left(\beta_{1}+\beta_{2}\right)(\eta-a)^{2}}{2 \bar{q}}+\frac{E_{1} \lambda_{1} \beta_{2}(\eta-a)(b-a)}{\bar{q}}\right. \\
& \left.\left.\left.\left.+\frac{E_{2} \lambda_{1} \lambda_{3}(\eta-a)\left[(b-a)^{2}-(\xi-a)^{2}\right]}{2 \bar{p}}+\frac{R E_{2}(b-a)}{\bar{p}}\right)\left(\frac{\lambda_{4} \mu_{1}\left[(b-a)^{2}-(\xi-a)^{2}\right]}{2 \bar{p}}\right)\right)\right]\right\} \\
& +\left(\phi(t)+\sigma_{1}|u|^{n_{1}}+\sigma_{2}|v|^{n_{2}}\right)\left\{\frac { 1 } { R } \left[\frac{\mu_{2}}{|\bar{q}|}\left(\frac{\lambda_{1}\left(\beta_{1}+\beta_{2}\right)(\eta-a)^{3}}{6}+\frac{\lambda_{1} \beta_{2}(\eta-a)(b-a)^{2}}{2}\right)\right.\right. \\
& +\frac{1}{E}\left(\left(\frac{E_{4} \alpha_{2}\left(\beta_{1}+\beta_{2}\right)(b-a)}{\bar{p}}+\frac{E_{3} \lambda_{1}\left(\beta_{1}+\beta_{2}\right)(\eta-a)^{2}}{2 \bar{q}}+\frac{E_{3} \lambda_{1} \beta_{2}(\eta-a)(b-a)}{\bar{q}}\right.\right. \\
& \left.+\frac{E_{4} \lambda_{1} \lambda_{3}(\eta-a)\left[(b-a)^{2}-(\xi-a)^{2}\right]}{2 \bar{p}}+\frac{R E_{4}(b-a)}{\bar{p}}\right)\left(\frac{\lambda_{2} \mu_{2}(\eta-a)^{2}}{2 \bar{q}}\right) \\
& +\left(\frac{E_{2} \alpha_{2}\left(\beta_{1}+\beta_{2}\right)(b-a)}{\bar{p}}+\frac{E_{1} \lambda_{1}\left(\beta_{1}+\beta_{2}\right)(\eta-a)^{2}}{\overline{2 q}}+\frac{E_{1} \lambda_{1} \beta_{2}(\eta-a)(b-a)}{\bar{q}}\right. \\
& \left.\left.\left.\left.+\frac{E_{2} \lambda_{1} \lambda_{3}(\eta-a)\left[(b-a)^{2}-(\xi-a)^{2}\right]}{2 \bar{p}}+\frac{R E_{2}(b-a)}{\bar{p}}\right)\left(\frac{\beta_{4} \mu_{2}(b-a)}{|q(b)|}\right)\right)\right]\right\},
\end{aligned}
$$

which, on taking the norm for $t \in[a, b]$, we have

$$
\left\|\mathcal{H}_{1}(u, v)\right\| \leq\left(\|\psi\|+\epsilon_{1}\|u\|^{m_{1}}+\epsilon_{2}\|v\|^{m_{2}}\right) \mathcal{D}_{1}+\left(\|\phi\|+\sigma_{1}\|u\|^{n_{1}}+\sigma_{2}\|v\|^{n_{2}}\right) \mathcal{D}_{2} .
$$

Similarly, we find that

$$
\left\|\mathcal{H}_{2}(u, v)\right\| \leq\left(\|\psi\|+\epsilon_{1}\|u\|^{m_{1}}+\epsilon_{2}\|v\|^{m_{2}}\right) \mathcal{D}_{3}+\left(\|\phi\|+\sigma_{1}\|u\|^{n_{1}}+\sigma_{2}\|v\|^{n_{2}}\right) \mathcal{D}_{4},
$$

where $\mathcal{D}_{i}(i=1, \ldots, 4)$ are given by (3.7). From the foregoing arguments, it follows that

$$
\|\mathcal{H}(u, v)\| \leq\left(\|\psi\|+\epsilon_{1}\|u\|^{m_{1}}+\epsilon_{2}\|v\|^{m_{2}}\right) \mathcal{E}_{1}+\left(\|\phi\|+\sigma_{1}\|u\|^{n_{1}}+\sigma_{2}\|v\|^{n_{2}}\right) \mathcal{E}_{2} \leq \varphi,
$$

where $\mathcal{E}_{1}$ and $\mathcal{E}_{2}$ are given by (3.5). Thus, we verify that $\mathcal{H}: \Theta \rightarrow \Theta$. 
Therefore, the operator $\mathcal{H}$ is completely continuous. Hence, by the Schauder fixed point theorem (3.2) there exist at least one solution for the problem (1.1) on $[a, b]$. The proof is now completed.

3.2. Uniqueness result. Here we establish the uniqueness of solutions for the given problem (1.1) by applying Banach's contraction mapping principle.

Theorem 3.4. Assume that:

$\left(S_{3}\right)$ For all $t \in[a, b]$ and $u_{i}, v_{i} \in \mathbb{R},(i=1,2)$, there exist $\ell_{i}>0,(i=1,2)$ such that:

$$
\left|f\left(t, u_{1}, v_{1}\right)-f\left(t, u_{2}, v_{2}\right)\right| \leq \ell_{1}\left(\left|u_{1}-u_{2}\right|+\left|v_{1}-v_{2}\right|\right)
$$

and

$$
\left|g\left(t, u_{1}, v_{1}\right)-g\left(t, u_{2}, v_{2}\right)\right| \leq \ell_{2}\left(\left|u_{1}-u_{2}\right|+\left|v_{1}-v_{2}\right|\right) .
$$

In addition, we suppose that

$$
\mathcal{E}_{1} \ell_{1}+\mathcal{E}_{2} \ell_{2}<1
$$

where $\mathcal{E}_{i}(i=1,2)$ are given by (3.5). Then, the problem (1.1) has a unique solution on $[a, b]$.

Proof. Firstly, we verify that $\mathcal{H} B_{\nu} \subset B_{\nu}$, where $B_{\nu}=\{(u, v) \in \mathcal{F} \times \mathcal{F}:\|(u, v)\| \leq \nu\}$. Define $Z_{1}=\sup _{t \in[a, b]}|f(t, 0,0)|$ and $Z_{2}=\sup _{t \in[a, b]}|g(t, 0,0)|$, and choose

$$
\nu \geq \frac{Z_{1} \mathcal{E}_{1}+Z_{2} \mathcal{E}_{2}}{1-\left(\ell_{1} \mathcal{E}_{1}+\ell_{2} \mathcal{E}_{2}\right)} .
$$

For any $(u, v) \in B_{\nu}, t \in[a, b]$, by condition $\left(S_{3}\right)$ we have

$$
\begin{aligned}
|f(t, u(t), v(t))| & =|f(t, u(t), v(t))-f(t, 0,0)+f(t, 0,0)| \\
& \leq|f(t, u(t), v(t))-f(t, 0,0)|+|f(t, 0,0)| \\
& \leq \ell_{1}(\|u\|+\|v\|)+Z_{1} \leq \ell_{1}\|(u, v)\|+Z_{1} \leq \ell_{1} \nu+Z_{1} .
\end{aligned}
$$

Similarly, $|g(t, u(t), v(t))| \leq \ell_{2}\|(u, v)\|+Z_{2} \leq \ell_{2} \nu+Z_{2}$. Then, for $(u, v) \in B_{\nu}$, we find that

$$
\begin{aligned}
& \left|\mathcal{H}_{1}(u, v)(t)\right| \\
\leq & \int_{a}^{t}\left(\frac{\mu_{1}}{|p(u)|} \int_{a}^{u}|f(z, u(z), v(z))| d z\right) d u \\
& +\frac{1}{R}\left[\alpha_{2}\left(\beta_{1}+\beta_{2}\right) \int_{a}^{b}\left(\frac{\mu_{1}}{|p(u)|} \int_{a}^{u}|f(z, u(z), v(z))| d z\right) d u\right. \\
& +\lambda_{1}\left(\beta_{1}+\beta_{2}\right) \int_{a}^{\eta} \int_{a}^{s}\left(\frac{\mu_{2}}{|q(u)|} \int_{a}^{u}|g(z, u(z), v(z))| d z\right) d u d s \\
& +\lambda_{1} \beta_{2}(\eta-a) \int_{a}^{b}\left(\frac{\mu_{2}}{|q(u)|} \int_{a}^{u}|g(z, u(z), v(z))| d z\right) d u \\
& \left.+\lambda_{1} \lambda_{3}(\eta-a) \int_{\xi}^{b} \int_{a}^{s}\left(\frac{\mu_{1}}{|p(u)|} \int_{a}^{u}|f(z, u(z), v(z))| d z\right) d u d s\right]
\end{aligned}
$$




$$
\begin{aligned}
& +\frac{1}{E R}\left[\left(E_{4} \alpha_{2}\left(\beta_{1}+\beta_{2}\right) \int_{a}^{b} \frac{1}{|p(z)|} d z+E_{3} \lambda_{1}\left(\beta_{1}+\beta_{2}\right) \int_{a}^{\eta} \int_{a}^{s} \frac{1}{|q(z)|} d z d s\right.\right. \\
& +E_{3} \lambda_{1} \beta_{2}(\eta-a) \int_{a}^{b} \frac{1}{|q(z)|} d z+E_{4} \lambda_{1} \lambda_{3}(\eta-a) \int_{\xi}^{b} \int_{a}^{s} \frac{1}{|p(z)|} d z d s \\
& \left.+R E_{4} \int_{a}^{t} \frac{1}{|p(z)|} d z\right)\left(\frac{\alpha_{4} \mu_{1}}{|p(b)|} \int_{a}^{b}|f(z, u(z), v(z))| d z\right)+\left(E_{4} \alpha_{2}\left(\beta_{1}+\beta_{2}\right) \int_{a}^{b} \frac{1}{|p(z)|} d z\right. \\
& +E_{3} \lambda_{1}\left(\beta_{1}+\beta_{2}\right) \int_{a}^{\eta} \int_{a}^{s} \frac{1}{|q(z)|} d z d s+E_{3} \lambda_{1} \beta_{2}(\eta-a) \int_{a}^{b} \frac{1}{|q(z)|} d z \\
& \left.+E_{4} \lambda_{1} \lambda_{3}(\eta-a) \int_{\xi}^{b} \int_{a}^{s} \frac{1}{|p(z)|} d z d s+R E_{4} \int_{a}^{t} \frac{1}{|p(z)|} d z\right) \\
& \times\left(\int_{a}^{\eta} \frac{\lambda_{2} \mu_{2}}{|q(s)|} \int_{a}^{s}|g(z, u(z), v(z))| d z d s\right)+\left(E_{2} \alpha_{2}\left(\beta_{1}+\beta_{2}\right) \int_{a}^{b} \frac{1}{|p(z)|} d z\right. \\
& +E_{1} \lambda_{1}\left(\beta_{1}+\beta_{2}\right) \int_{a}^{\eta} \int_{a}^{s} \frac{1}{|q(z)|} d z d s+E_{1} \lambda_{1} \beta_{2}(\eta-a) \int_{a}^{b} \frac{1}{|q(z)|} d z \\
& \left.+E_{2} \lambda_{1} \lambda_{3}(\eta-a) \int_{\xi}^{b} \int_{a}^{s} \frac{1}{|p(z)|} d z d s+R E_{2} \int_{a}^{t} \frac{1}{|p(z)|} d z\right)\left(\frac{\beta_{4} \mu_{2}}{|q(b)|} \int_{a}^{b}|g(z, u(z), v(z))| d z\right) \\
& +\left(E_{2} \alpha_{2}\left(\beta_{1}+\beta_{2}\right) \int_{a}^{b} \frac{1}{|p(z)|} d z+E_{1} \lambda_{1}\left(\beta_{1}+\beta_{2}\right) \int_{a}^{\eta} \int_{a}^{s} \frac{1}{|q(z)|} d z d s\right. \\
& +E_{1} \lambda_{1} \beta_{2}(\eta-a) \int_{a}^{b} \frac{1}{|q(z)|} d z+E_{2} \lambda_{1} \lambda_{3}(\eta-a) \int_{\xi}^{b} \int_{a}^{s} \frac{1}{|p(z)|} d z d s \\
& \left.\left.+R E_{2} \int_{a}^{t} \frac{1}{|p(z)|} d z\right)\left(\int_{\xi}^{b} \frac{\lambda_{4} \mu_{1}}{|p(s)|} \int_{a}^{s}|f(z, u(z), v(z))| d z d s\right)\right] \\
& \leq\left(\ell_{1} \nu+Z_{1}\right)\left\{\frac { 1 } { R } \left[\frac{\mu_{1}}{|\bar{p}|}\left(R \frac{(b-a)^{2}}{2}+\frac{\alpha_{2}\left(\beta_{1}+\beta_{2}\right)(b-a)^{2}}{2}+\frac{\lambda_{1} \lambda_{2}(\eta-a)\left[(b-a)^{3}-(\xi-a)^{3}\right]}{6}\right)\right.\right. \\
& +\frac{1}{E}\left(\left(\frac{E_{4} \alpha_{2}\left(\beta_{1}+\beta_{2}\right)(b-a)}{\bar{p}}+\frac{E_{3} \lambda_{1}\left(\beta_{1}+\beta_{2}\right)(\eta-a)^{2}}{2 q}+\frac{E_{3} \lambda_{1} \beta_{2}(\eta-a)(b-a)}{\bar{q}}\right.\right. \\
& \left.+\frac{E_{4} \lambda_{1} \lambda_{3}(\eta-a)\left[(b-a)^{2}-(\xi-a)^{2}\right]}{2 \bar{p}}+\frac{R E_{4}(b-a)}{\bar{p}}\right)\left(\frac{\alpha_{4} \mu_{1}(b-a)}{|p(b)|}\right) \\
& +\left(\frac{E_{2} \alpha_{2}\left(\beta_{1}+\beta_{2}\right)(b-a)}{\bar{p}}+\frac{E_{1} \lambda_{1}\left(\beta_{1}+\beta_{2}\right)(\eta-a)^{2}}{2 \bar{q}}+\frac{E_{1} \lambda_{1} \beta_{2}(\eta-a)(b-a)}{\bar{q}}\right. \\
& \left.\left.\left.\left.+\frac{E_{2} \lambda_{1} \lambda_{3}(\eta-a)\left[(b-a)^{2}-(\xi-a)^{2}\right]}{2 \bar{p}}+\frac{R E_{2}(b-a)}{\bar{p}}\right)\left(\frac{\lambda_{4} \mu_{1}\left[(b-a)^{2}-(\xi-a)^{2}\right]}{2 \bar{p}}\right)\right)\right]\right\} \\
& +\left(\ell_{2} \nu+Z_{2}\right)\left\{\frac { 1 } { R } \left[\frac{\mu_{2}}{|\bar{q}|}\left(\frac{\lambda_{1}\left(\beta_{1}+\beta_{2}\right)(\eta-a)^{3}}{6}+\frac{\lambda_{1} \beta_{2}(\eta-a)(b-a)^{2}}{2}\right)\right.\right. \\
& +\frac{1}{E}\left(\left(\frac{E_{4} \alpha_{2}\left(\beta_{1}+\beta_{2}\right)(b-a)}{\bar{p}}+\frac{E_{3} \lambda_{1}\left(\beta_{1}+\beta_{2}\right)(\eta-a)^{2}}{2 \bar{q}}+\frac{E_{3} \lambda_{1} \beta_{2}(\eta-a)(b-a)}{\bar{q}}\right.\right. \\
& \left.+\frac{E_{4} \lambda_{1} \lambda_{3}(\eta-a)\left[(b-a)^{2}-(\xi-a)^{2}\right]}{2 \bar{p}}+\frac{R E_{4}(b-a)}{\bar{p}}\right)\left(\frac{\lambda_{2} \mu_{2}(\eta-a)^{2}}{2 \bar{q}}\right) \\
& +\left(\frac{E_{2} \alpha_{2}\left(\beta_{1}+\beta_{2}\right)(b-a)}{\bar{p}}+\frac{E_{1} \lambda_{1}\left(\beta_{1}+\beta_{2}\right)(\eta-a)^{2}}{\overline{2 q}}+\frac{E_{1} \lambda_{1} \beta_{2}(\eta-a)(b-a)}{\bar{q}}\right.
\end{aligned}
$$




$$
\left.\left.\left.\left.+\frac{E_{2} \lambda_{1} \lambda_{3}(\eta-a)\left[(b-a)^{2}-(\xi-a)^{2}\right]}{2 \bar{p}}+\frac{R E_{2}(b-a)}{\bar{p}}\right)\left(\frac{\beta_{4} \mu_{2}(b-a)}{|q(b)|}\right)\right)\right]\right\},
$$

which implies

$$
\left\|\mathcal{H}_{1}(u, v)\right\| \leq\left(\ell_{1} \nu+Z_{1}\right) \mathcal{D}_{1}+\left(\ell_{2} \nu+Z_{2}\right) \mathcal{D}_{2}
$$

Similarly, we obtain $\left\|\mathcal{H}_{2}(u, v)\right\| \leq\left(\ell_{1} \nu+Z_{1}\right) \mathcal{D}_{3}+\left(\ell_{2} \nu+Z_{2}\right) \mathcal{D}_{4}$, where $\mathcal{D}_{i}(i=1, \ldots, 4)$ are defined by (3.7). which leads to the following:

$$
\|\mathcal{H}(u, v)\| \leq\left(\ell_{1} \nu+Z_{1}\right) \mathcal{E}_{1}+\left(\ell_{2} \nu+Z_{2}\right) \mathcal{E}_{2} \leq \nu .
$$

This shows that $\mathcal{H} B_{\nu} \subset B_{\nu}$.

Secondly, we show that the operator $\mathcal{H}$ is a contraction. For $\left(u_{1}, v_{1}\right),\left(u_{2}, v_{2}\right) \in$ $\mathcal{F} \times \mathcal{F}$, then

$$
\begin{aligned}
& \left|\mathcal{H}_{1}\left(u_{1}, v_{1}\right)(t)-\mathcal{H}_{1}\left(u_{2}, v_{2}\right)(t)\right| \\
& \leq \int_{a}^{t}\left(\frac{\mu_{1}}{|p(u)|} \int_{a}^{u}\left|f\left(z, u_{1}(z), v_{1}(z)\right)-f\left(z, u_{2}(z), v_{2}(z)\right)\right| d z\right) d u \\
& +\frac{1}{R}\left[\alpha_{2}\left(\beta_{1}+\beta_{2}\right) \int_{a}^{b}\left(\frac{\mu_{1}}{|p(u)|} \int_{a}^{u}\left|f\left(z, u_{1}(z), v_{1}(z)\right)-f\left(z, u_{2}(z), v_{2}(z)\right)\right| d z\right) d u\right. \\
& +\lambda_{1}\left(\beta_{1}+\beta_{2}\right) \int_{a}^{\eta} \int_{a}^{s}\left(\frac{\mu_{2}}{|q(u)|} \int_{a}^{u}\left|g\left(z, u_{1}(z), v_{1}(z)\right)-g\left(z, u_{2}(z), v_{2}(z)\right)\right| d z\right) d u d s \\
& +\lambda_{1} \beta_{2}(\eta-a) \int_{a}^{b}\left(\frac{\mu_{2}}{|q(u)|} \int_{a}^{u}\left|g\left(z, u_{1}(z), v_{1}(z)\right)-g\left(z, u_{2}(z), v_{2}(z)\right)\right| d z\right) d u \\
& \left.+\lambda_{1} \lambda_{3}(\eta-a) \int_{\xi}^{b} \int_{a}^{s}\left(\frac{\mu_{1}}{|p(u)|} \int_{a}^{u}\left|f\left(z, u_{1}(z), v_{1}(z)\right)-f\left(z, u_{2}(z), v_{2}(z)\right)\right| d z\right) d u d s\right] \\
& +\frac{1}{E R}\left[\left(E_{4} \alpha_{2}\left(\beta_{1}+\beta_{2}\right) \int_{a}^{b} \frac{1}{|p(z)|} d z+E_{3} \lambda_{1}\left(\beta_{1}+\beta_{2}\right) \int_{a}^{\eta} \int_{a}^{s} \frac{1}{|q(z)|} d z d s\right.\right. \\
& +E_{3} \lambda_{1} \beta_{2}(\eta-a) \int_{a}^{b} \frac{1}{|q(z)|} d z+E_{4} \lambda_{1} \lambda_{3}(\eta-a) \int_{\xi}^{b} \int_{a}^{s} \frac{1}{|p(z)|} d z d s \\
& \left.+R E_{4} \int_{a}^{t} \frac{1}{|p(z)|} d z\right)\left(\frac{\alpha_{4} \mu_{1}}{|p(b)|} \int_{a}^{b}\left|f\left(z, u_{1}(z), v_{1}(z)\right)-f\left(z, u_{2}(z), v_{2}(z)\right)\right| d z\right) \\
& +\left(E_{4} \alpha_{2}\left(\beta_{1}+\beta_{2}\right) \int_{a}^{b} \frac{1}{|p(z)|} d z+E_{3} \lambda_{1}\left(\beta_{1}+\beta_{2}\right) \int_{a}^{\eta} \int_{a}^{s} \frac{1}{|q(z)|} d z d s\right. \\
& +E_{3} \lambda_{1} \beta_{2}(\eta-a) \int_{a}^{b} \frac{1}{|q(z)|} d z+E_{4} \lambda_{1} \lambda_{3}(\eta-a) \int_{\xi}^{b} \int_{a}^{s} \frac{1}{|p(z)|} d z d s \\
& \left.+R E_{4} \int_{a}^{t} \frac{1}{|p(z)|} d z\right)\left(\int_{a}^{\eta} \frac{\lambda_{2} \mu_{2}}{|q(s)|} \int_{a}^{s}\left|g\left(z, u_{1}(z), v_{1}(z)\right)-g\left(z, u_{2}(z), v_{2}(z)\right)\right| d z d s\right) \\
& +\left(E_{2} \alpha_{2}\left(\beta_{1}+\beta_{2}\right) \int_{a}^{b} \frac{1}{|p(z)|} d z+E_{1} \lambda_{1}\left(\beta_{1}+\beta_{2}\right) \int_{a}^{\eta} \int_{a}^{s} \frac{1}{|q(z)|} d z d s\right. \\
& +E_{1} \lambda_{1} \beta_{2}(\eta-a) \int_{a}^{b} \frac{1}{|q(z)|} d z+E_{2} \lambda_{1} \lambda_{3}(\eta-a) \int_{\xi}^{b} \int_{a}^{s} \frac{1}{|p(z)|} d z d s \\
& \left.+R E_{2} \int_{a}^{t} \frac{1}{|p(z)|} d z\right)\left(\frac{\beta_{4} \mu_{2}}{|q(b)|} \int_{a}^{b}\left|g\left(z, u_{1}(z), v_{1}(z)\right)-g\left(z, u_{2}(z), v_{2}(z)\right)\right| d z\right)
\end{aligned}
$$




$$
\begin{aligned}
& +\left(E_{2} \alpha_{2}\left(\beta_{1}+\beta_{2}\right) \int_{a}^{b} \frac{1}{|p(z)|} d z+E_{1} \lambda_{1}\left(\beta_{1}+\beta_{2}\right) \int_{a}^{\eta} \int_{a}^{s} \frac{1}{|q(z)|} d z d s\right. \\
& +E_{1} \lambda_{1} \beta_{2}(\eta-a) \int_{a}^{b} \frac{1}{|q(z)|} d z+E_{2} \lambda_{1} \lambda_{3}(\eta-a) \int_{\xi}^{b} \int_{a}^{s} \frac{1}{|p(z)|} d z d s \\
& \left.\left.+R E_{2} \int_{a}^{t} \frac{1}{|p(z)|} d z\right)\left(\int_{\xi}^{b} \frac{\lambda_{4} \mu_{1}}{|p(s)|} \int_{a}^{s}\left|f\left(z, u_{1}(z), v_{1}(z)\right)-f\left(z, u_{2}(z), v_{2}(z)\right)\right| d z d s\right)\right] \\
& \leq \ell_{1}\left(\left|u_{1}-u_{2}\right|+\left|v_{1}-v_{2}\right|\right)\left\{\frac { 1 } { R } \left[\frac { \mu _ { 1 } } { | \overline { p } | } \left(R \frac{(b-a)^{2}}{2}+\frac{\alpha_{2}\left(\beta_{1}+\beta_{2}\right)(b-a)^{2}}{2}\right.\right.\right. \\
& \left.+\frac{\lambda_{1} \lambda_{2}(\eta-a)\left[(b-a)^{3}-(\xi-a)^{3}\right]}{6}\right) \\
& +\frac{1}{E}\left(\left(\frac{E_{4} \alpha_{2}\left(\beta_{1}+\beta_{2}\right)(b-a)}{\bar{p}}+\frac{E_{3} \lambda_{1}\left(\beta_{1}+\beta_{2}\right)(\eta-a)^{2}}{2 q}+\frac{E_{3} \lambda_{1} \beta_{2}(\eta-a)(b-a)}{\bar{q}}\right.\right. \\
& \left.+\frac{E_{4} \lambda_{1} \lambda_{3}(\eta-a)\left[(b-a)^{2}-(\xi-a)^{2}\right]}{2 \bar{p}}+\frac{R E_{4}(b-a)}{\bar{p}}\right)\left(\frac{\alpha_{4} \mu_{1}(b-a)}{|p(b)|}\right) \\
& +\left(\frac{E_{2} \alpha_{2}\left(\beta_{1}+\beta_{2}\right)(b-a)}{\bar{p}}+\frac{E_{1} \lambda_{1}\left(\beta_{1}+\beta_{2}\right)(\eta-a)^{2}}{2 \bar{q}}+\frac{E_{1} \lambda_{1} \beta_{2}(\eta-a)(b-a)}{\bar{q}}\right. \\
& \left.\left.\left.\left.+\frac{E_{2} \lambda_{1} \lambda_{3}(\eta-a)\left[(b-a)^{2}-(\xi-a)^{2}\right]}{2 \bar{p}}+\frac{R E_{2}(b-a)}{\bar{p}}\right)\left(\frac{\lambda_{4} \mu_{1}\left[(b-a)^{2}-(\xi-a)^{2}\right]}{2 \bar{p}}\right)\right)\right]\right\} \\
& +\ell_{2}\left(\left|u_{1}-u_{2}\right|+\left|v_{1}-v_{2}\right|\right)\left\{\frac { 1 } { R } \left[\frac{\mu_{2}}{|\bar{q}|}\left(\frac{\lambda_{1}\left(\beta_{1}+\beta_{2}\right)(\eta-a)^{3}}{6}+\frac{\lambda_{1} \beta_{2}(\eta-a)(b-a)^{2}}{2}\right)\right.\right. \\
& +\frac{1}{E}\left(\left(\frac{E_{4} \alpha_{2}\left(\beta_{1}+\beta_{2}\right)(b-a)}{\bar{p}}+\frac{E_{3} \lambda_{1}\left(\beta_{1}+\beta_{2}\right)(\eta-a)^{2}}{2 \bar{q}}+\frac{E_{3} \lambda_{1} \beta_{2}(\eta-a)(b-a)}{\bar{q}}\right.\right. \\
& \left.+\frac{E_{4} \lambda_{1} \lambda_{3}(\eta-a)\left[(b-a)^{2}-(\xi-a)^{2}\right]}{2 \bar{p}}+\frac{R E_{4}(b-a)}{\bar{p}}\right)\left(\frac{\lambda_{2} \mu_{2}(\eta-a)^{2}}{2 \bar{q}}\right) \\
& +\left(\frac{E_{2} \alpha_{2}\left(\beta_{1}+\beta_{2}\right)(b-a)}{\bar{p}}+\frac{E_{1} \lambda_{1}\left(\beta_{1}+\beta_{2}\right)(\eta-a)^{2}}{\overline{2 q}}+\frac{E_{1} \lambda_{1} \beta_{2}(\eta-a)(b-a)}{\bar{q}}\right. \\
& \left.\left.\left.\left.+\frac{E_{2} \lambda_{1} \lambda_{3}(\eta-a)\left[(b-a)^{2}-(\xi-a)^{2}\right]}{2 \bar{p}}+\frac{R E_{2}(b-a)}{\bar{p}}\right)\left(\frac{\beta_{4} \mu_{2}(b-a)}{|q(b)|}\right)\right)\right]\right\} .
\end{aligned}
$$

Thus, we have

$$
\left\|\mathcal{H}_{1}\left(u_{1}, v_{1}\right)-\mathcal{H}_{1}\left(u_{2}, v_{2}\right)\right\| \leq\left(\ell_{1} \mathcal{D}_{1}+\ell_{2} \mathcal{D}_{2}\right)\left(\left|u_{1}-u_{2}\right|+\left|v_{1}-v_{2}\right|\right) .
$$

In the same way, we find that

$$
\left\|\mathcal{H}_{2}\left(u_{1}, v_{1}\right)-\mathcal{H}_{2}\left(u_{2}, v_{2}\right)\right\| \leq\left(\ell_{1} \mathcal{D}_{3}+\ell_{2} \mathcal{D}_{4}\right)\left(\left|u_{1}-u_{2}\right|+\left|v_{1}-v_{2}\right|\right) .
$$

From (3.11) and (3.12), we have

$$
\left\|\mathcal{H}\left(u_{1}, v_{1}\right)-\mathcal{H}\left(u_{2}, v_{2}\right)\right\| \leq\left(\mathcal{E}_{1} \ell_{1}+\mathcal{E}_{2} \ell_{2}\right)\left(\left|u_{1}-u_{2}\right|+\left|v_{1}-v_{2}\right|\right),
$$

where $\mathcal{E}_{i}(i=1,2)$ are given by (3.5). So, by the assumption (3.10), the operator $\mathcal{H}$ is a contraction. Hence, by Banach contraction mapping principle the operator $\mathcal{H}$ has a unique fixed point, which implies that the problem (1.1) has a unique solution on $[a, b]$. This completes the proof . 


\section{ILLUSTRATIVE EXAMPLES}

In this section, we present aid examples to illustrate the main results that obtained:

Example 4.1. Consider the following coupled system of second-order ordinary differential equations

$$
\left\{\begin{array}{l}
\left(\left(\frac{1}{t+13}\right) u^{\prime}(t)\right)^{\prime}=\frac{3}{36}\left[\frac{e^{2 t}}{18}+\frac{|u(t)|}{25(1+|u(t)|)}+\frac{2}{17 \sqrt{t^{3}+4}} v(t)\right], t \in[0,3] \\
\left(\frac{8}{4 t^{2}+2 t+12} v^{\prime}(t)\right)^{\prime}==\frac{2}{93}\left[\frac{\cos t}{t+1}+\frac{\sqrt{t+1}}{3} u(t)+\frac{1}{t^{2}+5} v(t)\right], t \in[0,3],
\end{array}\right.
$$

supplemented with the following boundary conditions:

$$
\begin{aligned}
& \frac{7}{3} u(0)+\frac{5}{3} u(3)=\frac{1}{7} \int_{0}^{\frac{1}{2}} v(s) d s, \frac{4}{3} u^{\prime}(0)+u^{\prime}(3)=\frac{2}{7} \int_{0}^{\frac{1}{2}} v^{\prime}(s) d s \\
& \frac{1}{9} v(0)+\frac{2}{9} v(3)=\frac{3}{7} \int_{\frac{5}{2}}^{3} u(s) d s, \frac{3}{9} v^{\prime}(0)+\frac{4}{9} v^{\prime}(3)=\frac{4}{7} \int_{\frac{5}{2}}^{3} u^{\prime}(s) d s .
\end{aligned}
$$

Here

$$
\begin{gathered}
p(t)=\frac{1}{t+13} \quad \text { and } \quad q(t)=\left(\frac{8}{4 t^{2}+2 t+12}\right) \\
\mu_{1}=3 / 36, \mu_{2}=2 / 93, a=0, b=3, \eta=1 / 2, \quad \xi=5 / 2 \\
\lambda_{1}=1 / 7, \quad \lambda_{2}=2 / 7, \quad \lambda_{3}=3 / 7, \lambda_{4}=4 / 7 \\
\alpha_{1}=7 / 3, \alpha_{2}=5 / 3, \alpha_{3}=4 / 3, \alpha_{4}=1 \\
\beta_{1}=1 / 9, \quad \beta_{2}=2 / 9, \quad \beta_{3}=3 / 9, \beta_{4}=4 / 9
\end{gathered}
$$

Using the given data,we find that $|R| \approx 1.323129 \neq 0,|E| \approx 115.6354 \neq 0$ $(R$ and $E$ is given by $(2.4)), \bar{p} \approx 0.0625, \bar{q}=0.148148, \mathcal{D}_{1} \approx 17.1389708, \mathcal{D}_{2} \approx$ $0.06036034, \mathcal{D}_{3} \approx 38.2023705, \mathcal{D}_{4} \approx 4.565128967,\left[\bar{p}, \bar{q}\right.$ and $\mathcal{D}_{i}(i=1, \ldots, 4)$ are defined in $(3.7)], \mathcal{E}_{1} \approx 17.19933114$ and $\mathcal{E}_{2} \approx 42.76749946\left[\mathcal{E}_{1}\right.$ and $\mathcal{E}_{2}$ are given by (3.5)]. Here,

$$
|f(t, u, v)| \leq \frac{1}{216}+\frac{1}{300}\|u\|+\frac{1}{204}\|v\|,|g(t, u, v)| \leq \frac{2}{465}+\frac{2}{279}\|u\|+\frac{2}{465}\|v\|,
$$

with, $k_{0}=1 / 216, k_{1}=1 / 300, k_{2}=1 / 204, \hat{k}_{0}=2 / 465, \hat{k}_{1}=2 / 279, \hat{k}_{2}=2 / 465$.

Morever, we obtain $\mathcal{E}_{1} k_{1}+\mathcal{E}_{2} \hat{k}_{1} \approx 0.3639081609<1, \mathcal{E}_{1} k_{2}+\mathcal{E}_{2} \hat{k}_{2} \approx 0.268256681<1$. By Theorem (3.2) the problem (4.1) and (4.2) has at least one solution on [0,3]. 
Example 4.2. Consider the following system:

$$
\left\{\begin{array}{l}
\left(\left(\frac{1}{t+13}\right) u^{\prime}(t)\right)^{\prime}=\frac{1}{44}\left[\frac{2}{t^{3}-15}+\left(t+\frac{9}{36 \sqrt{t+2}}\right)\left[(u(t))^{\frac{3}{4}}+(v(t))^{\frac{2}{5}}\right]\right], t \in[0,3], \\
\left(\frac{8}{4 t^{2}+2 t+12} v^{\prime}(t)\right)^{\prime}=\frac{3}{42}\left[\frac{3}{7} t^{2}+\left(t^{2}\right)\left[(u(t))^{\frac{1}{2}}+(v(t))^{\frac{3}{8}}\right]\right], t \in[0,3],
\end{array}\right.
$$

subject to the coupled boundary conditions of Example 4.1.

With $m_{1}=3 / 4 m_{2}=2 / 5, n_{1}=1 / 2, n_{2}=3 / 8$. It's clear, that the condition $\left(S_{2}\right)$ is verified. Thus, by the conclusion of Theorem 3.3 to the system (4.3) with boundary conditions (4.2) there exist a solution of the problem (4.3) with the boundary conditions (4.2) on $[0,3]$.

Example 4.3. Consider the following system:

$$
\left\{\begin{array}{l}
\left(\frac{1}{t+13} u^{\prime}(t)\right)^{\prime}=\frac{1}{36}\left[\frac{|u(t)|}{1+|u(t)|}+\frac{1}{\sqrt{t^{3}+1}} v(t)+3 \sin (t)\right], t \in[0,3] \\
\left(\sqrt{2 t^{2}+12} v^{\prime}(t)\right)^{\prime}=\frac{1}{93}\left[\frac{4}{t^{2}+4} u(t)+\tan ^{-1} v(t)-5 t\right], t \in[0,3]
\end{array}\right.
$$

with the coupled boundary conditions (4.2).

Observe that

$$
\left|f\left(t, u_{1}, v_{1}\right)-f\left(t, u_{2}, v_{2}\right)\right| \leq \frac{1}{36}\left(\left|u_{1}-u_{2}\right|+\left|v_{1}-v_{2}\right|\right)
$$

and

$$
\left|g\left(t, u_{1}, v_{1}\right)-g\left(t, u_{2}, v_{2}\right)\right| \leq \frac{1}{93}\left(\left|u_{1}-u_{2}\right|+\left|v_{1}-v_{2}\right|\right)
$$

which implies that $\ell_{1}=1 / 36, \ell_{2}=1 / 93$. By using the same data in example (4.1), we have

$$
\mathcal{E}_{1} \approx 17.19933114, \mathcal{E}_{2} \approx 42.76749946 \text { and } \mathcal{E}_{1} \ell_{1}+\mathcal{E}_{2} \ell_{2} \approx 0.937624784<1 .
$$

Hence by Theorem 3.4, the problem (4.4) with the coupled boundary conditions (4.2) has a unique solution on $[0,3]$.

\section{CONCLUSIONS}

In this paper, we have derived the existence and uniqueness results for a selfadjoint coupled system of nonlinear ordinary differential equations supplemented with nonlocal non-separated integral boundary conditions on an arbitrary domain. We have applied the standard tools of the fixed-point theory to establish the desired results. Our results are new and significantly contribute to the literature on nonlinear nonlocal integral boundary value problems involving self-adjoint ordinary differential equations. 


\section{ACKNOWLEDGMENT}

This project was funded by the Deanship of Scientific Research (DSR), King Abdulaziz University, Jeddah, Saudi Arabia under grant no. (KEP-MSc-63-130-42). The authors, therefore, acknowledge with thanks DSR technical and financial support.

\section{REFERENCES}

[1] B. Ahmad, A. Alsaedi, N. Al-Malki, On higher-order nonlinear boundary value problems with nonlocal multipoint integral boundary conditions, Lithuanian Math. J. 56 (2016), 143-163.

[2] B. Ahmad, A. Alsaedi, M. Alsulami, Existence theory for coupled nonlinear third-order ordinary differential equations with nonlocal multi-point anti-periodic type boundary conditions on an arbitrary domain, AIMS Mathematics, 4 (2019), 1634-1663.

[3] F.T. Akyildiz, H. Bellout, K. Vajravelu, R.A. Van Gorder, Existence results for third order nonlinear boundary value problems arising in nano boundary layer fluid flows over stretching surfaces, Nonlinear Anal. Real World Appl. 12 (2011), 2919-2930.

[4] J. Brüning, V. Geyler, K. Pankrashkin, Spectra of self-adjoint extensions and applications to solvable Schrödinger operators, Rev. Math. Phys. 20 (2008), 1-70.

[5] S. Clark, J. Henderson, Uniqueness implies existence and uniqueness criterion for non local boundary value problems for third-order differential equations, Proc. Amer. Math. Soc. 134 (2006), 3363-3372.

[6] Y. F. Dolgii, Application of self-adjoint boundary value problems to investigation of stability of periodic delay systems, Proc. Steklov Inst. Math. (2006), Control, Stability, and Inverse Problems of Dynamics, Suppl. 2, S16-S25.

[7] P.W. Eloe, B. Ahmad, Positive solutions of a nonlinear $n$th order boundary value problem with nonlocal conditions, Appl. Math. Lett. 18 (2005), 521-527.

[8] M. Feng, X. Zhang, W. Ge, Existence theorems for a second order nonlinear differential equation with nonlocal boundary conditions and their applications, J. Appl. Math. Comput. 33 (2010), 137-153.

[9] J.R. Graef, J.R.L. Webb, Third order boundary value problems with nonlocal boundary conditions, Nonlinear Anal. 71 (2009), 1542-1551.

[10] A. Granas, J. Dugundji, Fixed Point Theory, Springer-Verlag, New York, 2005.

[11] M. Greguš, F. Neumann, F.M. Arscott, Three-point boundary value problems in differential equations, Proc. London Math. Soc. 3 (1964), 459-470.

[12] M.R. Grossinho, F.M. Minhos, Existence result for some third order separated boundary value problems. Proceedings of the Third World Congress of Nonlinear Analysts, Part 4 (Catania, 2000), Nonlinear Anal. 47 (2001), 2407-2418.

[13] C.P. Gupta, Solvability of a three-point nonlinear boundary value problem for a second order ordinary differential equations, J. Math. Anal. Appl. 168 (1998), 540-551.

[14] V.A. Il'in, E.I. Moiseev, A nonlocal boundary value problem of the first kind for the SturmLiouville operator in differential and difference interpretations (Russian), Differentsialnye Uravneniya 23 (1987), 1198-1207.

[15] I.Y. Karaca, F.T. Fen, Positive solutions of $n$ th-order boundary value problems with integral boundary conditions, Math. Model. Anal. 20 (2015), 188-204. 
[16] S.K. Ntouyas, Nonlocal Initial and Boundary Value Problems: A survey, In Handbook on Differential Equations: Ordinary Differential Equations, Edited by A. Canada, P. Drabek and A. Fonda, Elsevier Science B. V., 2005, 459-555.

[17] H.M. Srivastava, S.K. Ntouyas, M. Alsulami, A. Alsaedi, B. Ahmad, A self-adjoint coupled system of nonlinear ordinary differential equations with nonlocal multi-point boundary conditions on an arbitrary domain, Appl. Sci. 2021, 11, 4798.

[18] J. Sugie, Interval criteria for oscillation of second-order self-adjoint impulsive differential equations. Proc. Am. Math. Soc. 2020, 148, 1095-1108.

[19] Y. Sun, L. Liu, J. Zhang, R.P. Agarwal, Positive solutions of singular three-point boundary value problems for second-order differential equations, J. Comput. Appl. Math. 230 (2009), $738-750$.

[20] A.A. Vladimirov, Variational principles for self-adjoint Hamiltonian systems (Russian), Mat. Zametki 107 (2020), 633-636.

[21] L. Wang, M. Pei, W. Ge, Existence and approximation of solutions for nonlinear second-order four-point boundary value problems, Math. Comput. Model. 50 (2009), 1348-1359.

[22] J.R.L. Webb, G. Infante, Positive solutions of nonlocal boundary value problems: A unified approach, J. London Math. Soc. 74 (2006), 673-693.

[23] L. Zheng, X. Zhang, Modeling and Analysis of Modern Fluid Problems, Mathematics in Science and Engineering, Elsevier (Academic Press), London, 2017. 